\title{
Diastereoselectivity studies on the photo-activated cycloaddition of 5-(1,2-dioxyethyl)-2(5H)-furanones to alkenes
}

\author{
Joan R. Cucarull-González, Ramon Alibés, Marta Figueredo,* and Josep Font \\ Universitat Autónoma de Barcelona, Departament de Química, 08193, Bellaterra, Spain \\ E-mail: ramon.alibes@uab.es, marta.figueredo@uab.es
}

This article is dedicated to Prof Michael Orfanopoulos on the occasion of his $67^{\text {th }}$ birthday

DOI: http://dx.doi.org/10.3998/ark.5550190.0016.314

\begin{abstract}
A series of 2(5H)-furanones, bearing a 1,2-dioxyethyl substituent at the $\gamma$-carbonyl position, have been prepared and explored as substrates in photochemical reactions with alkenes. Compared to the simpler oxymethyl analogues, the homologation of the side chain is highly beneficial to the antifacial selectivity of the [2+2] cycloadditions. Most reactions occur in synthetically useful yields, giving access to new polyfunctionalized cyclobutane-fused furanones.
\end{abstract}

Keywords: Furanones, photochemistry, [2+2] cycloaddition, diastereoselectivity

\section{Introduction}

There are many natural products, which incorporate in their substructure a $2(5 H)$-furanone subunit. ${ }^{1}$ Many of these compounds display a variety of biological activities and have thus attracted the interest of synthetic organic chemists. The furanone ring is also present in some unnatural drugs including antifungal, antibacterial and anti-inflammatory agents. Moreover, several chiral substituted $2(5 H)$-furanones, which are readily available in enantiomerically pure form from chiral pool materials, have been used as the starting substrates for the preparation of an assortment of targeted compounds of challenging structures with diverse complexity and potential utility. Among them, we focused our attention on several compounds with a polysubstituted cyclobutane framework ${ }^{2-4}$ and over the years, we have developed enantioselective synthetic approaches to various pheromones ${ }^{5-10}$ and cyclobutane nucleoside analogues, ${ }^{11-13}$ some of them built on a 3-oxabicyclo[3.2.1] heptane scaffold. In these syntheses, the cyclobutane core was generated through a photo-activated [2+2] cycloaddition of a $2(5 \mathrm{H})$ furanone derivative to ethylene or another alkene. ${ }^{14}$ 
The [2+2] photocycloaddition is in fact one of the processes more extensively applied to generate cyclobutane rings ${ }^{15-18}$ and a critical aspect is the control of the reaction stereochemistry. Previously, we used 5-oxymethyl-2(5H)-furanones 1, as the starting substrates (Scheme 1) and we verified that the attack of ethylene occurs preferentially by the face opposite to the substituent of the furanone, although the degree of facial discrimination was influenced by the nature of this substituent, steric and electronic factors being at play. ${ }^{19-22}$ Among the studied substrates, the pivaloyl derivative 1a displayed the higher antifacial selectivity, although the presence of a vinylic methyl group, $\mathbf{1 b}$, was detrimental to the diastereoselectivity, increasing the amount of the isomer derived from the competitive synfacial pathway.

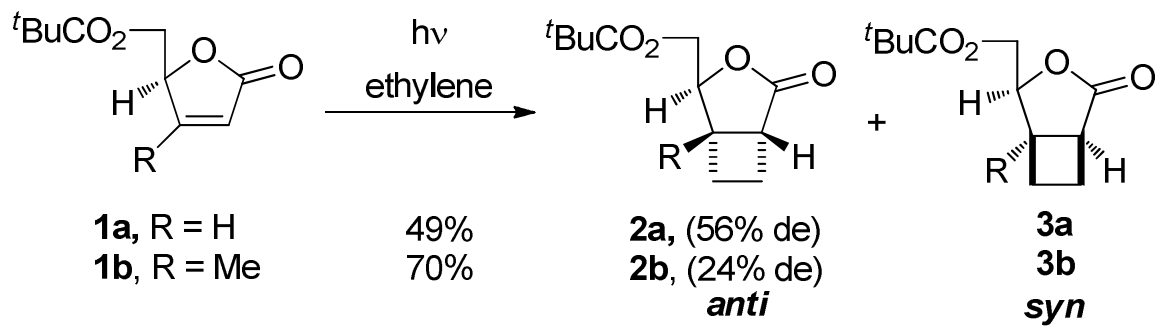

Scheme 1. Photocycloaddition of furanones 1a-b to ethylene.

In further studies on the $C_{2}$-symmetric bislactones 4 (Figure 1), we found higher degrees of antifacial selectivity in their photoreactions with ethylene even when methyl groups were attached to the $\beta$-carbonyl position, and we observed that the protective groups of the central diol unit had a noticeable influence on the diastereofacial selectivity, which is almost complete with the TMS protection. ${ }^{23}$ Unfortunately, the elaborated preparation of these lactones restricts their synthetic application. For this reason, we considered of interest to explore the performance of the more accessible 2(5H)-furanones $\mathbf{5}$, bearing also a 1,2-dioxyethyl unit as the substituent at the $\gamma$-carbonyl position, as substrates in photochemical reactions with alkenes. To the best of our knowledge, there is no report dealing with [2+2] photocycloadditions of these oxymethyl homologues of $\mathbf{1}$. We anticipated that the facial selectivity of their cycloadditions could be significantly improved in respect to that of lactones $\mathbf{1}$, provided that a favorable combination of steric and electronic factors diminished the accessibility of the syn face. Moreover, lactones $\mathbf{5}$ were visualized as interesting chiral synthons with good opportunities for subsequent diastereoselective transformations. In this article we describe the preparation of lactones $\mathbf{5 a - h}$ and their $[2+2]$ photocycloadditions to alkenes. 


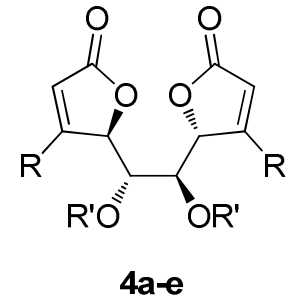

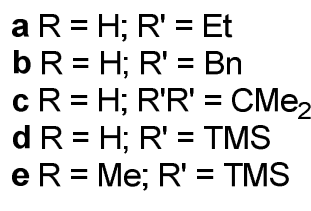
d $R=H ; R^{\prime}=T M S$
e $R=M e ; R^{\prime}=T M S$

$4 a-e$

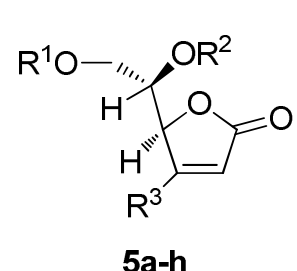

5a-h

$$
\begin{aligned}
& \text { a } \mathrm{R}^{1}=\mathrm{Bn} ; \mathrm{R}^{2}=\mathrm{H} ; \mathrm{R}^{3}=\mathrm{H} \\
& \text { b } R^{1}=B n ; R^{2}=C^{t} B u ; R^{3}=H \\
& \text { c } \mathrm{R}^{1}=\mathrm{Bn} ; \mathrm{R}^{2}=\mathrm{Bz} ; \mathrm{R}^{3}=\mathrm{H} \\
& \text { d } \mathrm{R}^{1}=\mathrm{Bn} ; \mathrm{R}^{2}=\mathrm{TBDMS} ; \mathrm{R}^{3}=\mathrm{H} \\
& \text { e } \mathrm{R}^{1}=\mathrm{CO}^{t} \mathrm{Bu} ; \mathrm{R}^{2}=\mathrm{H} ; \mathrm{R}^{3}=\mathrm{H} \\
& \text { f } \mathrm{R}^{1}=\mathrm{CO}^{t} \mathrm{Bu} ; \mathrm{R}^{2}=\mathrm{CO}^{t} \mathrm{Bu} ; \mathrm{R}^{3}=\mathrm{H} \\
& \text { g R }^{1}=\mathrm{CO}^{t} \mathrm{Bu} ; \mathrm{R}^{2}=\mathrm{CO}^{i} \mathrm{Bu} ; \mathrm{R}^{3}=\mathrm{H} \\
& \text { h } \mathrm{R}^{1}=\mathrm{CO}^{t} \mathrm{Bu} ; \mathrm{R}^{2}=\mathrm{CO}^{t} \mathrm{Bu} ; \mathrm{R}^{3}=\mathrm{Me}
\end{aligned}
$$

Figure 1. Bislactones 4 previously studied and 5-(1,2-dioxyethyl)-2(5H)-furanones $\mathbf{5}$ used in the present study.

\section{Results and Discussion}

Benzyl and pivaloyl were chosen as the protective groups of the primary alcohol. Benzyl was selected because it was expected that an aromatic residue could be involved into a beneficial $\pi$ stacking interaction with the carbon-carbon double bond of the lactone, shielding the syn face more effectively and hence preventing the approach of the alkene. On the other hand, the pivaloyl group had previously displayed the better diasteroselectivity in the former type 1 lactone series. The secondary hydroxyl was either unprotected or derivatized to a sterically demanding group.

Lactone 5a was prepared from (+)-dimethyl L-tartrate by a previously described procedure ${ }^{24}$ and it was then converted into the new lactones $\mathbf{5 b}, \mathbf{5 c}$ and $\mathbf{5 d}$, following standard methodologies. The synthesis of the pivaloyl derivative 5e was accomplished through a similar sequence to that described for $\mathbf{5 a}$ (Scheme 2). Thus, (+)-dimethyl L-tartrate, $\mathbf{6}$, was transformed into 2,3-O-isopropylidene L-threitol (7) by a described procedure which involves acetalization followed by reduction. ${ }^{25}$ After several attempts of monopivaloylation of the symmetric diol $\mathbf{7}$, the best regioselectivity was obtained by absorbing the diol over silica gel and treating a suspension of this silica gel in hexane with pivaloyl chloride and pyridine. ${ }^{26}$ Under these conditions, the starting diol was recovered in part and the monopivaloate 8 was isolated in $41 \%$ yield $(61 \%$ over consumed 7 ), along with a minor quantitiy of the dipivaloyl derivative (15\% yield). The alcohol $\mathbf{8}$ was then subjected to the Swern oxidation to furnish the corresponding aldehyde, which without any purification was reacted with $\mathrm{Ph}_{3} \mathrm{P}=\mathrm{CHCO}_{2} \mathrm{Me}$ in dry methanol, ${ }^{27}$ delivering a 7:1 mixture of the expected $(Z)$ - and $(E)-\alpha, \beta$-unsaturated esters. On treatment with methanolic hydrogen chloride, this mixture afforded the targeted lactone $5 \mathbf{e}$ in $51 \%$ yield for the three steps, along with the $(E)-\alpha, \beta$-unsaturated ester $\mathbf{9}$ in $7 \%$ yield. Lactones $\mathbf{5 f}$ and $\mathbf{5 g}$ were prepared from $\mathbf{5 e}$ following standard procedures. Finally, the furanone $\mathbf{5 h}$, bearing a methyl group at the $\beta$-carbonyl position, was prepared by treatment of $\mathbf{5 f}$ with diazomethane, followed by pyrolysis of the corresponding pyrazoline $\mathbf{1 0}$ in refluxing 1,4-dioxane, in overall $55 \%$ yield (Scheme 3). 


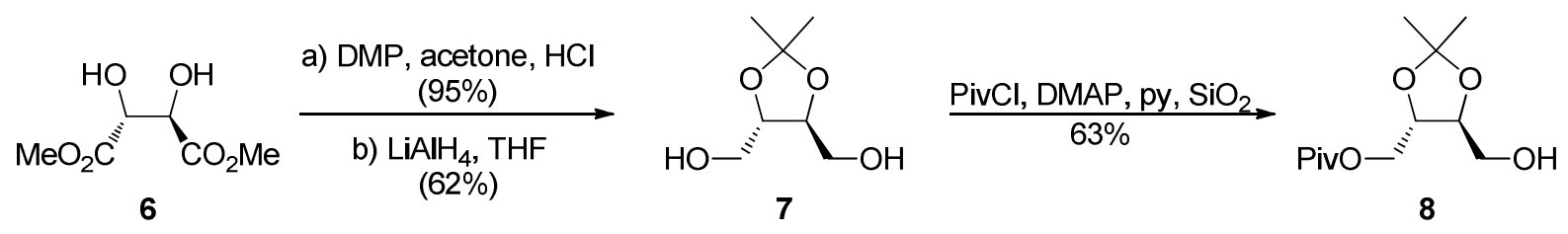

a) Swern oxidation

b) $\mathrm{Ph}_{3} \mathrm{P}=\mathrm{CHCO}_{2} \mathrm{Me}, \mathrm{MeOH}$

c) $\mathrm{HCl}(3 \mathrm{M}), \mathrm{MeOH}$

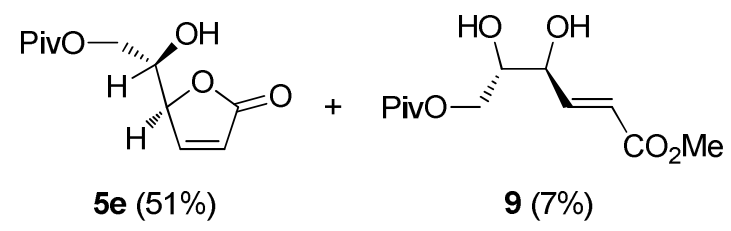

Scheme 2. Preparation of the pivaloyl derivative 5e.

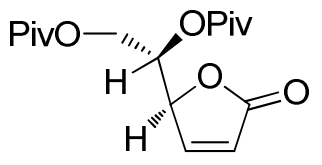

$5 \mathbf{f}$

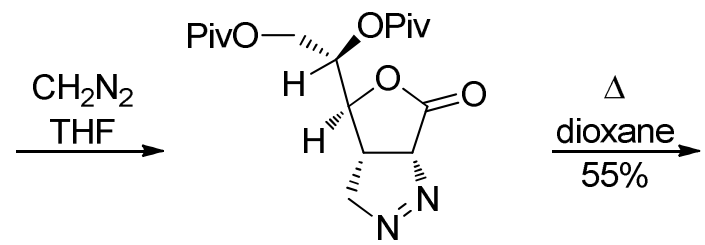

10<smiles>CC1=CC(=O)O[C@H]1[C@@H](CO[Na])O[Na]</smiles>

$5 h$

Scheme 3. Preparation of the $\beta$-methyl derivative $\mathbf{5 h}$.

For the photochemical study, the furanones 5a-h in acetone solutions saturated with ethylene were irradiated through a Pyrex vessel with a $125 \mathrm{~W}$ high-pressure mercury lamp at $-20{ }^{\circ} \mathrm{C}$ (Table 1). The progress of the cycloaddition was monitored by GC and the irradiation was prolonged until complete conversion of the starting furanone. The cycloadducts anti 11a-h and syn 12a-h were then purified through silica gel column chromatography and individually characterized.

The photocycloaddition of 5a to ethylene delivered the two expected cyclobutane diastereomers 11a and 12a in good yield with a very good degree of antifacial selectivity (Table 1 , entry 1). Introduction of the bulky pivaloyl group at the secondary alcohol as in the reference substrate 1a diminished the rate of the cycloaddition and did not produce a substantial improvement of the facial discrimination (Table 1, entry 2). This could be in agreement with a greater influence of the expected $\pi$ stacking interaction with the participation of the benzyl group (Figure 2, A) over the steric barrier exerted by the pivaloyl residue. Nevertheless, the free hydroxyl group in 5a can enable intramolecular hydrogen bonding with the carbonyl oxygen of the lactone (Figure 2, B) that can lead to very efficient hindrance of the syn face. The photoreaction of the benzoyl derivative (entry 3) was faster but occurred with lower facial selectivity. This observation argues against the $\pi$ stacking hypothesis but a competitive interaction between the two aromatic rings in this particular substrate cannot be totally discarded (Figure 2, C). In agreement with the precedents, the TBDMS derivative 5d (entry 4) displayed 
the highest diastereoselectivity within this series, delivering exclusively the anti cycloadduct 11d, although in slightly lower yield, despite the complete consumption of the starting furanone. The cycloadduct yields within the primary pivaloyl series of furanones were also good (Table 1, entries 5-7), with very good diastereoselectivities for the diester derivatives $\mathbf{5 f}$ and $\mathbf{5 g}$ and somewhat lower for the substrate bearing the secondary free alcohol 5e. Apparently, the size of the acyl group does not play a decisive role in the stereochemical outcome of the reaction. We conclude that, in the photocycloadditon to ethylene, the efficiency of the process in all the cases is superior to that previously found for 1a, both in terms of yield and antifacial selectivity. To evaluate the influence of a methyl group attached to the $\beta$-carbonyl position on these new substrates, lactone $\mathbf{5 h}$ was irradiated under the same conditions (Table 1, entry 8). This reaction delivered the corresponding cycloadducts $11 \mathrm{~h}$ and $\mathbf{1 2 h}$ in good yield although the anti:syn selectivity decreased, as it has been observed for the parallel process from $\mathbf{1 b}$.

Table 1. Photocycloaddition of lactones 5a-h to ethylene<smiles>[R]OC[C@]1([2H])OC(=O)C=C1[R]</smiles>

5a-h
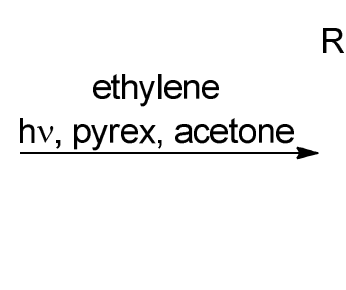

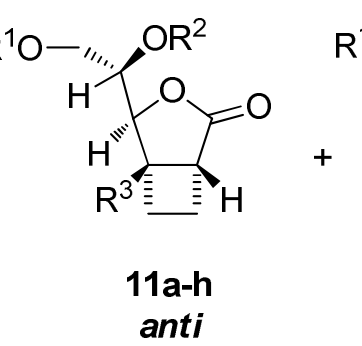

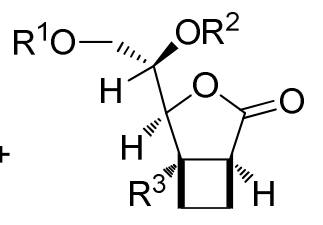

12a-h

\begin{tabular}{ccccccccc}
\hline Entry & Furanone & $\mathrm{R}^{1}$ & $\mathrm{R}^{2}$ & $\mathrm{R}^{3}$ & $\begin{array}{c}\text { Time } \\
(\mathrm{min})\end{array}$ & $\begin{array}{c}\text { Yield }^{a} \\
(\%)\end{array}$ & anti-11:syn-12 $^{b}$ & $\begin{array}{c}\text { de } \\
(\%)\end{array}$ \\
\hline 1 & $\mathbf{5 a}$ & $\mathrm{Bn}$ & $\mathrm{H}$ & $\mathrm{H}$ & 130 & 78 & $8: 1$ & 78 \\
2 & $\mathbf{5 b}$ & $\mathrm{Bn}$ & $\mathrm{CO}^{t} \mathrm{Bu}$ & $\mathrm{H}$ & 320 & 74 & $9: 1$ & 80 \\
3 & $\mathbf{5 c}$ & $\mathrm{Bn}$ & $\mathrm{Bz}$ & $\mathrm{H}$ & 95 & 70 & $4: 1$ & 60 \\
4 & $\mathbf{5 d}$ & $\mathrm{Bn}$ & $\mathrm{TBDMS}$ & $\mathrm{H}$ & 210 & 58 & only anti-11d & 100 \\
5 & $\mathbf{5 e}$ & $\mathrm{CO}^{t} \mathrm{Bu}$ & $\mathrm{H}$ & $\mathrm{H}$ & 365 & $78^{c}$ & $6: 1$ & 72 \\
6 & $\mathbf{5 f}$ & $\mathrm{CO}^{t} \mathrm{Bu}$ & $\mathrm{CO}^{t} \mathrm{Bu}$ & $\mathrm{H}$ & 400 & 77 & $9: 1$ & 80 \\
7 & $\mathbf{5 g}$ & $\mathrm{CO}^{t} \mathrm{Bu}$ & $\mathrm{CO}^{i} \mathrm{Bu}$ & $\mathrm{H}$ & 300 & 83 & $9: 1$ & 80 \\
8 & $\mathbf{5 h}$ & $\mathrm{CO}^{t} \mathrm{Bu}$ & $\mathrm{CO}^{t} \mathrm{Bu}$ & $\mathrm{Me}$ & 300 & $80^{d}$ & $4: 1$ & 60 \\
\hline
\end{tabular}

${ }^{a}$ Yield of isolated product as a mixture of stereoisomers after column chromatography purification. ${ }^{b}$ Isomer ratio from ${ }^{1} \mathrm{H}$ NMR and GC analysis of the of the isolated mixture of products. ${ }^{c} 10 \%$ of starting $\mathbf{5 e}$ was recovered. ${ }^{d} 11 \%$ of starting $\mathbf{5 h}$ was recovered. 
A

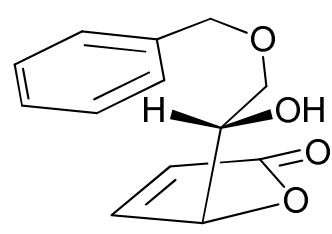

$5 a$
B

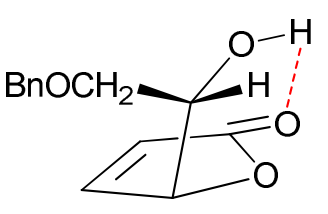

$5 a$
C

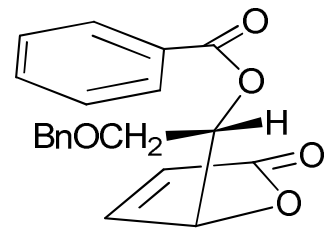

5c

Figure 2. Conformers of 5a with $\pi$-stacking interaction (A) or intramolecular hydrogen bonding (B) and conformer of $\mathbf{5 c}$ with $\pi$-stacking interaction involving the benzoyl group (C).

The structural elucidation of the new cyclobutanes was supported by NMR analysis of pure isolated samples, including mono- and bidimensional experiments. Once the signal corresponding to the protons attached to C-4 and C-5 are identified, the value of the coupling constant between these two protons $J_{4,5}$ can be used as a reliable diagnostic for assigning the anti/syn relative configuration. ${ }^{5,7-9}$ Thus, for the anti isomers $\mathbf{1 1}$ the $J_{4,5}$ values were in the range from 0 to $2.3 \mathrm{~Hz}$, while for the syn isomers 12 oscillate between 5.2 and $5.7 \mathrm{~Hz}$ (Table 2). These coupling constants were determined on the signals corresponding to $\mathrm{H}-4$, because in most cases the signal of H-5 overlapped with other cyclobutane protons. Unfortunately, for the TBDMS derivative 11d, the signal of $\mathrm{H}-4$ is also masked by that of the benzyl protons and, hence, $J_{4,5}$ could not be determined, but we assumed that the only product isolated from the photocycloaddition of $\mathbf{5 d}$ should have the anti configuration. The stereochemical assignment of the isomers $\mathbf{1 1 h} / \mathbf{1 2 h}$, lacking the proton at $\mathrm{C}-5$, was deduced from the ${ }^{13} \mathrm{C}$ chemical shift of the $\beta$-methyl group, which is more sterically compressed in the anti isomer (17.5 ppm) compared to the syn (22.0 ppm).

Previously, we developed an alternative entry to fused cyclobutane furanones that avoided the use of ethylene ${ }^{28}$ and we decided to explore this option also on the new substrates. As such, lactones 5a and 5e were irradiated in acetonitrile solutions containing an excess of (Z)-1,2dichloroethylene and, without isolating the individual isomers, the product mixture of dichlorocyclobutanes was reduced by treatment with tributyltin hydride and AIBN in THF (Scheme 4). This protocol applied to 5e gave a 7:1 mixture of 11e and 12e in 67\% overall yield. Hence, the antifacial selectivity is similar to that obtained with ethylene from the same substrate (Table 1, entry 5) and, although the total yield is slightly lower, this procedure may have practical advantages when working on a larger scale. Unfortunately, the same protocol applied to 5a did not lead to any identifiable products. 
Table 2. Significant NMR data of compounds 11 and 12

\begin{tabular}{|c|c|c|c|c|c|}
\hline \multirow[b]{2}{*}{ Compound } & \multicolumn{4}{|c|}{$\begin{array}{c}11 \\
\text { anti }\end{array}$} & $\begin{array}{c}12 \\
\text { syn }\end{array}$ \\
\hline & $\mathrm{R}^{1}$ & $\mathrm{R}^{2}$ & $\delta \mathrm{H}-4$ & $\delta \mathrm{H}-5$ & $J_{4,5}(\mathrm{~Hz})$ \\
\hline $11 a$ & $\mathrm{Bn}$ & $\mathrm{H}$ & 4.38 & $\approx 3.1$ & 2.3 \\
\hline $12 a$ & $\mathrm{Bn}$ & $\mathrm{H}$ & 4.47 & $\approx 3.1$ & 5.2 \\
\hline 11b & $\mathrm{Bn}$ & $\mathrm{CO}^{t} \mathrm{Bu}$ & 4.70 & $\approx 3.0$ & $2.0 / 1.1$ \\
\hline $12 \mathrm{~b}$ & $\mathrm{Bn}$ & $\mathrm{CO}^{t} \mathrm{Bu}$ & 4.72 & $\approx 3.1$ & 5.3 \\
\hline 11c & $\mathrm{Bn}$ & $\mathrm{Bz}$ & 4.80 & $\approx 3.0$ & 0.8 \\
\hline $12 \mathrm{c}$ & $\mathrm{Bn}$ & $\mathrm{Bz}$ & 4.88 & $\approx 3.0$ & 5.6 \\
\hline $11 \mathrm{e}$ & $\mathrm{CO}^{t} \mathrm{Bu}$ & $\mathrm{H}$ & 4.40 & $\approx 3.1$ & 2.2 \\
\hline $12 \mathrm{e}$ & $\mathrm{CO}^{t} \mathrm{Bu}$ & $\mathrm{H}$ & 4.44 & $\approx 3.2$ & 5.4 \\
\hline $11 f$ & $\mathrm{CO}^{t} \mathrm{Bu}$ & $\mathrm{CO}^{t} \mathrm{Bu}$ & 4.58 & $\approx 3.0$ & $2.2 / 1.0$ \\
\hline $12 f$ & $\mathrm{CO}^{t} \mathrm{Bu}$ & $\mathrm{CO}^{t} \mathrm{Bu}$ & 4.58 & $\approx 3.2$ & 5.2 \\
\hline $11 \mathrm{~g}$ & $\mathrm{CO}^{t} \mathrm{Bu}$ & $\mathrm{CO}^{i} \mathrm{Bu}$ & 4.55 & $\approx 3.0$ & $2.3 / 1.0$ \\
\hline $12 \mathrm{~g}$ & $\mathrm{CO}^{t} \mathrm{Bu}$ & $\mathrm{CO}^{i} \mathrm{Bu}$ & 4.68 & $\approx 2.5$ & 5.7 \\
\hline
\end{tabular}
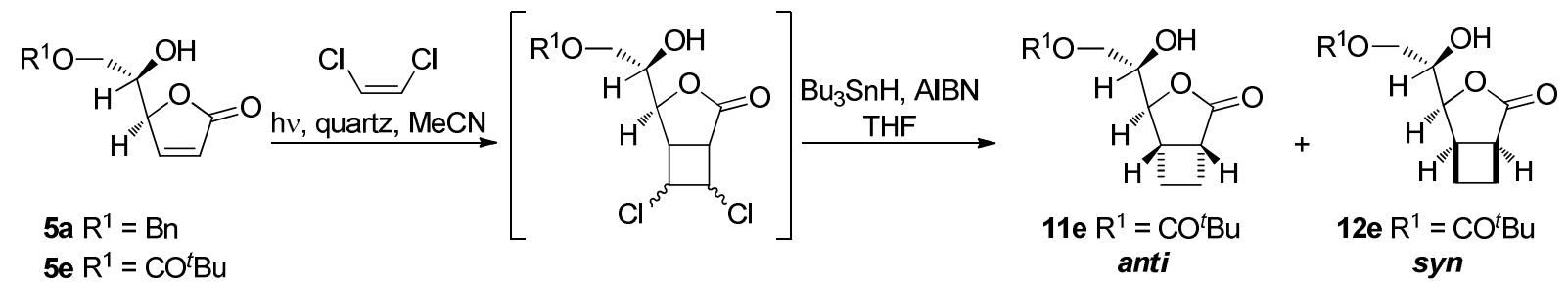

Scheme 4. Photocycloaddition of 5a and 5e to (Z)-1,2-dichloroethylene and subsequent reductive treatment.

To broaden the synthetic applicability of the cycloadducts, we investigated the photocycloaddition of $\mathbf{5 b}$ and $\mathbf{5 f}$ to 1,1-diethoxyethylene (Scheme 5). In contrast to the previous reactions, this cycloaddition is amenable to produce regioisomers, depending on the head to tail (HT) or head to head $(\mathrm{HH})$ orientation of the two reagents. Moreover, each orientation may occur, as before, through an anti- or syn-facial approach, overall producing up to four isomers. In previous studies with 1a and other similar lactones, we observed that, compared to acetonitrile, the less polar solvents favored the HT regioisomer and decreased the antifacial selectivity. ${ }^{29}$ The photocycloadditions of lactones $\mathbf{5 b}$ and $\mathbf{5 f}$ were assayed in acetonitrile, diethyl ether and hexane, in the presence of an excess of 1,1-diethoxyethylene (Table 3). In all these solvents, the 
irradiation of $\mathbf{5 b}$ was completely regioselective, furnishing exclusively the HT adducts $\mathbf{1 3 b}$ and 14b in moderate yields (Table 3, entries 1-3). Conversely, in the irradiation of lactone $\mathbf{5 f}$ under identical conditions (Table 3, entries 4-6), the HH-anti cycloadduct $\mathbf{1 5 f}$ was detected as a minor product, with the higher proportion in acetonitrile as the solvent, as expected. The antifacial selectivity was quite similar in all the cases, not being influenced by the solvent polarity.

Table 3. Photocycloaddition of lactones $\mathbf{5 b}$ and $\mathbf{5 f}$ to 1,1-diethoxyethylene

\begin{tabular}{cccccccc}
\hline Entry & Furanone & Solvent & $\begin{array}{c}\text { Time } \\
(\min )\end{array}$ & $\begin{array}{c}\text { Yield }^{a} \\
(\%)\end{array}$ & $\mathbf{1 3 : 1 4 : 1 5 : 1 6}{ }^{b}$ & HT:HH & anti: $\boldsymbol{s y n}$ \\
\hline 1 & $\mathbf{5 b}$ & $\mathrm{MeCN}$ & 120 & 54 & $79: 21:-:-$ & only HT & $4: 1$ \\
2 & $\mathbf{5 b}$ & $\mathrm{Et}_{2} \mathrm{O}$ & 300 & 44 & $76: 24:-:-$ & only HT & $3: 1$ \\
3 & $\mathbf{5 b}$ & hexane & 120 & 61 & $79: 21:-:-$ & only HT & $4: 1$ \\
4 & $\mathbf{5 f}$ & $\mathrm{MeCN}$ & 210 & 81 & $64: 16: 20:-$ & $4: 1$ & $5: 1$ \\
5 & $\mathbf{5 f}$ & $\mathrm{Et}_{2} \mathrm{O}$ & 150 & 41 & $75: 21: 4:-$ & $24: 1$ & $4: 1$ \\
6 & $\mathbf{5 f}$ & hexane & 270 & 74 & $75: 21: 4:-$ & $24: 1$ & $4: 1$ \\
\hline
\end{tabular}

${ }^{a}$ Yield of isolated product as a mixture of stereoisomers after column chromatography purification. ${ }^{b}$ Isomer ratio from GC analysis of the isolated mixture of products.

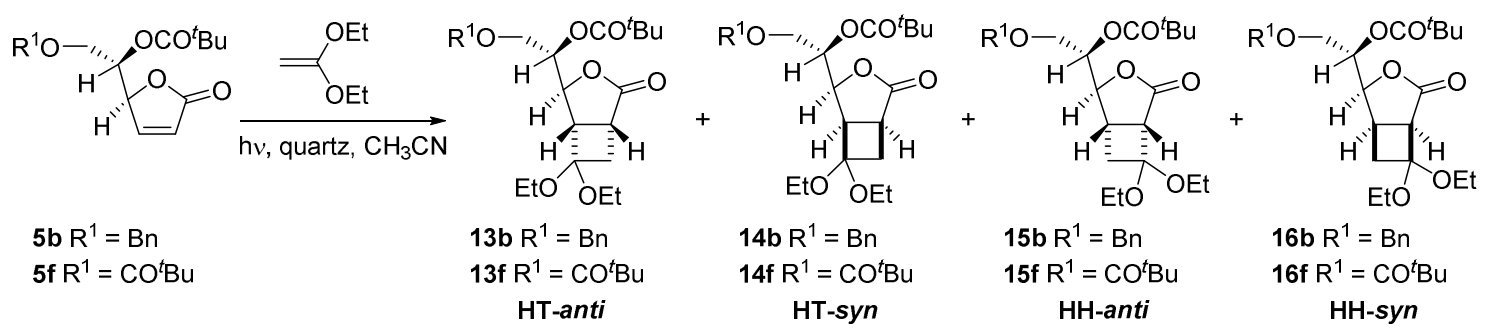

Scheme 5. Photocycloaddition of $\mathbf{5 b}$ and $\mathbf{5 f}$ to 1,1-diethoxyethylene.

\section{Conclusions}

A series of 2(5H)-furanones 5 bearing a 1,2-dioxyethyl unit as the substituent at the $\gamma$-carbonyl position were prepared and explored as substrates in photochemical reactions with alkenes compared to the simpler oxymethyl analogues $\mathbf{1}$. The additional oxymethyl fragment was highly beneficial to the antifacial selectivity of the [2+2] cycloadditions to ethylene, reaching in most cases diastereomeric excesses around $80 \%$. Furthermore, (Z)-dichloroethylene, as a solid surrogate of ethylene, was also used to prepare the same cyclobutane products in a more practical way. The photoreaction of lactone $\mathbf{5 b}$ to 1,1-diethoxyethylene showed a complete regioselectivity towards the head to tail orientation, independently of the solvent, while that of $\mathbf{5 f}$ was less regioselective and solvent dependent. For this alkene, the antifacial selectivity was lower 
than that observed for ethylene. Most reactions occurred in synthetically useful yields, giving access to new polyfunctionalized cyclobutane-fused furanones that may be further elaborated to natural or unnatural cyclobutanes of interest.

\section{Experimental Section}

General. Unless otherwise noted, analytical grade solvents and commercially available reagents were used without further purification. Solvents were purified and dried by standard procedures. The solutions were concentrated using a rotary evaporator at 15-20 Torr. Analytical thin layer chromatography (TLC) was performed on $0.25 \mathrm{~mm}$ silica gel $60-\mathrm{F}$ plates and visualized by ultraviolet irradiation and $\mathrm{KMnO}_{4}$ stains. Gas chromatography (GC) analysis was performed using a cross-linked capillary column with 5\% dimethylsilicone. Flash column chromatography (FCC) was carried out on silica gel (230-400 mesh). Melting points were determined at the hot stage and are uncorrected. Optical rotations were measured on a Propol Automatisches Dr Kermchem polarimeter. ${ }^{1} \mathrm{H}$ NMR spectra were recorded at 250 or $360 \mathrm{MHz}$ and ${ }^{13} \mathrm{C} \mathrm{NMR}$ spectra at 62.5 or $90 \mathrm{MHz}$ in $\mathrm{CDCl}_{3}$ solutions unless otherwise indicated at the Servei de Ressonància Magnètica Nuclear de la Universitat Autònoma de Barcelona. Microanalyses, Mass Spectrometry analysis, and IR spectra were performed at the Servei d'Anàlisi Química de la Universitat Autònoma de Barcelona. Compound 5a was prepared following a literature procedure. $^{24}$

(5S)-5-[(1S)-2-Benzyloxy-1-pivaloyloxyethyl]-2(5H)-furanone (5b). Pivaloyl chloride (0.53 $\mathrm{mL}, 4.30 \mathrm{mmol})$ was added to an ice-cooled solution of $5 \mathbf{a}(500 \mathrm{mg}, 2.13 \mathrm{mmol})$ and dry pyridine $(1 \mathrm{~mL})$ in dry $\mathrm{CH}_{2} \mathrm{Cl}_{2}(5 \mathrm{~mL})$ under a nitrogen atmosphere. The mixture was stirred overnight as it came to room temperature. The solvent was removed and the resulting oily residue was purified by FCC (hexanes/EtOAc, 4:1) to afford the title compound $\mathbf{5 b}$ as a colorless oil (585 mg, 86\%): $[\alpha]_{\mathrm{D}}-74$ (c 2.8, $\mathrm{CHCl}_{3}$ ). IR (ATR): 3090, 3064, 3031, 2970, 2870, 1759, $1729,1603 \mathrm{~cm}^{-1} .{ }^{1} \mathrm{H}$ NMR $\left(250 \mathrm{MHz}, \mathrm{CDCl}_{3}\right) \delta_{\mathrm{H}} 7.39\left(\mathrm{dd}, J_{4,3} 5.7 \mathrm{~Hz}, J_{4,5} 2.2 \mathrm{~Hz}, 1 \mathrm{H}, \mathrm{H}-4\right)$, 7.55-7.15 (m, 5H, H-Ar), 6.12 (dd, $\left.J_{3,4} 5.7 \mathrm{~Hz}, J_{3,5} 1.6 \mathrm{~Hz}, 1 \mathrm{H}, \mathrm{H}-3\right), 5.42-5.34$ (m, 1H, H-5), 5.26 (ddd, J 7.2, 6.0, $2.5 \mathrm{~Hz}, 1 \mathrm{H}, \mathrm{CHO}), 4.64\left(\mathrm{~d}, J_{\text {gem }} 11.4 \mathrm{~Hz}, 1 \mathrm{H}, \underline{\mathrm{C}}_{2} \mathrm{Ph}\right), 4.58$ (d, $J_{\text {gem }} 11.4$ $\mathrm{Hz}, 1 \mathrm{H}, \mathrm{CH}_{2} \mathrm{Ph}$ ), 3.82 (dd, J 9.7, 7.2 Hz, $1 \mathrm{H}, \mathrm{CH}_{2} \mathrm{O}$ ), 3.69 (dd, J 9.7, $6.0 \mathrm{~Hz}, 1 \mathrm{H}, \mathrm{CH}_{2} \mathrm{O}$ ), 1.14 (s, $9 \mathrm{H}, t$-Bu). ${ }^{13} \mathrm{C} \mathrm{NMR}\left(62.5 \mathrm{MHz}, \mathrm{CDCl}_{3}\right) \delta_{\mathrm{C}} 177.2(\mathrm{C}=\mathrm{O}), 172.1(\mathrm{C}=\mathrm{O}), 152.6(\mathrm{C}-4), 137.2$, 128.3, 127.7, 127.4 (C-Ar), 122.2 (C-3), 81.3 (C-5), $73.4\left(\mathrm{CH}_{2} \mathrm{O}\right), 68.9(\mathrm{CHO}), 67.8\left(\mathrm{CH}_{2} \mathrm{Ph}\right)$, $38.6\left(\mathrm{Me}_{3} \underline{\mathrm{C}}\right), 26.7\left(\underline{\mathrm{Me}}_{3} \mathrm{C}\right)$. MS (ESI) $\mathrm{m} / z(\%): 319.1\left(\mathrm{M}+\mathrm{H}^{+}, 2\right), 341.1\left(\mathrm{M}+\mathrm{Na}^{+}, 20\right), 357.1$ $\left(\mathrm{M}+\mathrm{K}^{+}, 100\right)$. Anal. Calcd. for $\mathrm{C}_{18} \mathrm{H}_{22} \mathrm{O}_{5}: \mathrm{C}, 67.91 ; \mathrm{H}, 6.97$. Found: $\mathrm{C}, 67.57 ; \mathrm{H}, 7.02 \%$.

(5S)-5-[(1S)-1-Benzoyloxy-2-benzyloxyethyl]-2(5H)-furanone (5c). Benzoyl chloride $(0.5 \mathrm{~mL}$, $4.31 \mathrm{mmol})$ was added dropwise to an ice-cooled solution of $\mathbf{5 a}(502 \mathrm{mg}, 2.14 \mathrm{mmol})$ and pyridine $(1 \mathrm{~mL})$ in dry $\mathrm{CH}_{2} \mathrm{Cl}_{2}(5 \mathrm{~mL})$ under a nitrogen atmosphere. The mixture was stirred overnight as it came to room temperature. The solvent was removed and the resulting oil was 
purified by FCC (hexanes/EtOAc, 4:1) to give the title compound 5c as a colorless oil (664 mg, 92\%): $[\alpha]_{\mathrm{D}}-58$ (c 2.8, $\mathrm{CHCl}_{3}$ ). IR (ATR): 3031, 3062, 2922, 1718, 1758, $1600 \mathrm{~cm}^{-1} .{ }^{1} \mathrm{H} \mathrm{NMR}$ $\left(250 \mathrm{MHz}, \mathrm{CDCl}_{3}\right) \delta_{\mathrm{H}} 7.97-7.93(\mathrm{~m}, 2 \mathrm{H}, \mathrm{H}-\mathrm{Ar}), 7.60$ (tt, J 7.2, $\left.1.2 \mathrm{~Hz}, 1 \mathrm{H}, \mathrm{H}-\mathrm{Ar}\right), 7.50-7.30$ (m, 8H, H-Ar, 1H, H-4), 6.12 (dd, $\left.J_{3,4} 6.2 \mathrm{~Hz}, J_{3,5} 2.0 \mathrm{~Hz}, 1 \mathrm{H}, \mathrm{H}-3\right)$, 5.56-5.46 (m, 2H, H-5, CHO), $4.67\left(\mathrm{~d}, J_{\text {gem }} 12.0 \mathrm{~Hz}, 1 \mathrm{H}, \mathrm{C}_{2} \mathrm{Ph}\right), 4.60\left(\mathrm{~d}, J_{\text {gem }} 12.0 \mathrm{~Hz}, 1 \mathrm{H}, \mathrm{C}_{2} \mathrm{Ph}\right), 3.95(\mathrm{dd}, J 12.0,6.9 \mathrm{~Hz}$, $\left.1 \mathrm{H}, \mathrm{CH}_{2} \mathrm{O}\right), 3.84\left(\mathrm{dd}, J 12.0,5.6 \mathrm{~Hz}, 1 \mathrm{H}, \mathrm{CH}_{2} \mathrm{O}\right) .{ }^{13} \mathrm{C} \mathrm{NMR}\left(62.5 \mathrm{MHz}, \mathrm{CDCl}_{3}\right) \delta_{\mathrm{C}} 172.8(\mathrm{C}=\mathrm{O})$, $165.9(\mathrm{C}=\mathrm{O}), 153.2(\mathrm{C}-4), 137.8,134.0,130.5,130.2$, 129.4, 128.9, 128.4, 128.2 (C-Ar), 123.2 (C-3), 82.0 (C-5), $74.1\left(\mathrm{CH}_{2} \mathrm{O}\right), 70.7(\mathrm{CHO}), 68.5\left(\underline{\mathrm{CH}}_{2} \mathrm{Ph}\right)$. MS (ESI) $\mathrm{m} / z(\%): 361.1\left(\mathrm{M}+\mathrm{Na}^{+}\right.$, 15), $377.1\left(\mathrm{M}+\mathrm{K}^{+}, 100\right)$. HRMS $(\mathrm{FAB}+)$ : calculated for $\left[\mathrm{C}_{20} \mathrm{H}_{18} \mathrm{O}_{5}+\mathrm{H}\right]^{+}$339.1232; found, 339.1224.

(5S)-5-[(1S)-2-Benzyloxy-1-tert-butyldimethyilsilyloxyethyl]-2(5H)-furanone $\quad(5 \mathrm{~d}) . \quad$ To a stirred and ice-cooled solution of 5a $(533 \mathrm{mg}, 2.28 \mathrm{mmol})$ in dry $\mathrm{CH}_{2} \mathrm{Cl}_{2}(10 \mathrm{~mL})$ under a nitrogen atmosphere were added a solution of imidazole $(449 \mathrm{mg}, 6.60 \mathrm{mmol})$ in dry $\mathrm{CH}_{2} \mathrm{Cl}_{2}(3$ $\mathrm{mL})$ and a solution of tert-butyldimethylsilyl chloride $(986 \mathrm{mg}, 6.54 \mathrm{mmol})$ in dry $\mathrm{CH}_{2} \mathrm{Cl}_{2}(2$ $\mathrm{mL}$ ). The mixture was stirred at room temperature overnight. The solvent was evaporated and the resulting residue was purified by FCC (hexanes/EtOAc, from 9:1 to 4:1) to afford the title compound $5 \mathbf{d}(752 \mathrm{mg}, 95 \%)$ as a colorless oil: $[\alpha]_{\mathrm{D}}-58.5\left(c \mathrm{cos}, \mathrm{CHCl}_{3}\right)$. IR (ATR): 2928, 2856, 1788, 1754, $1471 \mathrm{~cm}^{-1} .{ }^{1} \mathrm{H}$ NMR $\left(360 \mathrm{MHz}, \mathrm{CDCl}_{3}\right) \delta_{\mathrm{H}} 7.41\left(\mathrm{dd}, J_{4,3} 5.7 \mathrm{~Hz}, J_{4,5} 1.6 \mathrm{~Hz}\right.$, 1H, H-4), 7.39-7.29 (m, 5H, H-Ar), 6.12 (dd, $J_{3,4} 5.7$ Hz, $J_{3,5} 2.0 \mathrm{~Hz}, 1 \mathrm{H}, \mathrm{H}-3$ ), 5.19 (broad s, $1 \mathrm{H}, \mathrm{H}-5), 4.57$ (d, $\left.J_{\text {gem }} 11.8 \mathrm{~Hz}, 1 \mathrm{H}, \mathrm{C}_{2} \mathrm{Ph}\right), 4.52$ (d, $J_{\text {gem }} 11.8 \mathrm{~Hz}, 1 \mathrm{H}, \mathrm{C}_{2} \mathrm{Ph}$ ), 4.02 (ddd, J 6.7, 5.3, 3.7 Hz, 1H, CHO), $3.62\left(\mathrm{dd}, J 9.6,6.7 \mathrm{~Hz}, 1 \mathrm{H}, \mathrm{CH}_{2} \mathrm{O}\right), 3.51\left(\mathrm{dd}, J\right.$ 9.6, $5.3 \mathrm{~Hz}, 1 \mathrm{H}, \mathrm{CH}_{2} \mathrm{O}$ ), $0.86(\mathrm{~s}, 9 \mathrm{H}, t-\mathrm{Bu}), 0.06\left(\mathrm{~s}, 6 \mathrm{H}, \mathrm{SiMe}_{2}\right) .{ }^{13} \mathrm{C} \mathrm{NMR}\left(90.0 \mathrm{MHz}, \mathrm{CDCl}_{3}\right) \delta_{\mathrm{C}} 173.1(\mathrm{C}=\mathrm{O}), 154.0(\mathrm{C}-$ 4), 137.7, 128.5, 127.8, 127.7 (C-Ar), 122.4 (C-3), $84.0(\mathrm{C}-5), 73.6\left(\mathrm{CH}_{2} \mathrm{Ph}\right), 71.2\left(\mathrm{CH}_{2} \mathrm{O}\right), 70.9$ (CHO), $25.7\left(\underline{\mathrm{Me}}_{3} \mathrm{C}\right), 18.0\left(\mathrm{Me}_{3} \underline{\mathrm{C}}\right),-4.6$ (SiMe), -4.9 (SiMe). HRMS (FAB+): calculated for $\left[\mathrm{C}_{19} \mathrm{H}_{28} \mathrm{O}_{4} \mathrm{Si}+\mathrm{H}\right]^{+} 371.1649$; found, 371.1647 .

4- $\boldsymbol{O}$-Pivaloyl-2,3- $\boldsymbol{O}$-isopropylidene-L-threitol (8). Silica gel $(10 \mathrm{~g})$ was added to a solution of 2,3-O-isopropylidene L-threitol (7) $(10.19 \mathrm{~g}, 62.8 \mathrm{mmol})$ in $\mathrm{CH}_{2} \mathrm{Cl}_{2}(500 \mathrm{~mL})$ and the suspension was stirred vigorously for $10 \mathrm{~min}$. The solvent was removed and hexane $(1 \mathrm{~L})$ and pyridine $(10$ $\mathrm{mL}$ ) were added. The suspension was cooled at $0{ }^{\circ} \mathrm{C}$ and pivaloyl chloride $(7.5 \mathrm{~mL}, 60.8 \mathrm{mmol})$ was added dropwise under a nitrogen atmosphere. The resulting suspension was stirred overnight at room temperature. Then, it was placed in a chromatography column and washed with $\mathrm{CH}_{2} \mathrm{Cl}_{2}$ until all the organic material was eluted. The solvent was evaporated and the resulting oil was purified by FCC (from hexanes/EtOAc 1:1 to EtOAc) to afford the following fractions: (i) the dipivaloyl derivative $8 \mathbf{a}(2.04 \mathrm{~g}, 10 \%)$; (ii) the monopivaloate 8 (6.34 g, 41\%); and (iii) starting material 7 (3.37 g, 33\%).

8. $[\alpha]_{\mathrm{D}}+24.1$ ( c 2.1, $\mathrm{CHCl}_{3}$ ). IR (ATR): $3492,2982,2936,2876,1731,1397 \mathrm{~cm}^{-1} .{ }^{1} \mathrm{H} \mathrm{NMR}$ $\left(250 \mathrm{MHz}, \mathrm{CDCl}_{3}\right): \delta_{\mathrm{H}} 4.25-4.15(\mathrm{~m}, 2 \mathrm{H}, 2 \mathrm{H}-1), 4.15-4.05(\mathrm{~m}, 1 \mathrm{H}, \mathrm{H}-2), 3.93\left(\mathrm{ddd}, J_{3,2} 8.0 \mathrm{~Hz}\right.$, $\left.J_{3,4} 4.4 \mathrm{~Hz}, J_{3,4} 3.7 \mathrm{~Hz}, 1 \mathrm{H}, \mathrm{H}-3\right), 3.80$ (dd, $\left.J_{\text {gem }} 11.9 \mathrm{~Hz}, J_{4,3} 3.7 \mathrm{~Hz}, 1 \mathrm{H}, \mathrm{H}-4\right), 3.65$ (dd, $J_{\text {gem }}$ $\left.11.9 \mathrm{~Hz}, J_{4,3} 4.4 \mathrm{~Hz}, 1 \mathrm{H}, \mathrm{H}-4\right), 2.10(\mathrm{~s}, 1 \mathrm{H}, \mathrm{OH}), 1.45$ (s, 6H, $\left.\mathrm{Me}_{2} \mathrm{C}\right), 1.24(\mathrm{~s}, 9 \mathrm{H}, t-\mathrm{Bu})$. ${ }^{13} \mathrm{C}$ NMR $\left(62.5 \mathrm{MHz}, \mathrm{CDCl}_{3}\right) \delta_{\mathrm{C}} 178.8(\mathrm{C}=\mathrm{O}), 110.0\left(\mathrm{Me}_{2} \underline{\mathrm{C}}\right) ; 78.7(\mathrm{C}-2), 75.4(\mathrm{C}-3), 64.0(\mathrm{C}-4)$, 
$62.2(\mathrm{C}-1), 39.3\left(\mathrm{Me}_{3} \underline{\mathrm{C}}\right), 27.6(\mathrm{Me}) . \mathrm{MS}(\mathrm{EI}) \mathrm{m} / \mathrm{z}(\%): 231\left(\mathrm{M}^{+}-15,45\right)$. Anal. Calcd. for $\mathrm{C}_{12} \mathrm{H}_{22} \mathrm{O}_{5}$ : C, 58.52; $\mathrm{H}, 9.00$; found: $\mathrm{C}, 58.51 ; \mathrm{H}, 8.97 \%$.

8a. $[\alpha]_{\mathrm{D}}+0.9$ (c 2.2, $\left.\mathrm{CHCl}_{3}\right)$. IR (ATR): 2972, 2933, 2873, 1731, $1397 \mathrm{~cm}^{-1} .{ }^{1} \mathrm{H}$ NMR (250 $\left.\mathrm{MHz}, \mathrm{CDCl}_{3}\right) \delta_{\mathrm{H}} 4.28\left(\mathrm{ddd}, J_{\text {gem }} 11.9 \mathrm{~Hz}, J_{1,2}=J_{4,3} 2.7 \mathrm{~Hz}, J_{1,3}=J_{4,2} 1.4 \mathrm{~Hz}, 2 \mathrm{H}, \mathrm{H}-1, \mathrm{H}-4\right), 4.18$ (ddd, $\left.J_{\text {gem }} 11.9 \mathrm{~Hz}, J_{1,2}=J_{4,3} 2.7 \mathrm{~Hz}, J_{1,3}=J_{4,2} 1.4 \mathrm{~Hz}, 2 \mathrm{H}, \mathrm{H}-1, \mathrm{H}-4\right), 4.08-4.04$ (m, 2H, H-2, H-3), $1.41\left(\mathrm{~s}, 6 \mathrm{H}, \mathrm{O}_{2} \mathrm{CMe}_{2}\right), 1.20(\mathrm{~s}, 18 \mathrm{H}, 2 \times t-\mathrm{Bu}) .{ }^{13} \mathrm{C} \mathrm{NMR}\left(62.5 \mathrm{MHz}, \mathrm{CDCl}_{3}\right) \delta_{\mathrm{C}} 177.7(\mathrm{C}=\mathrm{O})$, $109.5\left(\mathrm{Me}_{2} \mathrm{C}\right), 75.6$ (C-2, C-3), 63.0 (C-1, C-4), $38.5\left(\mathrm{Me}_{3} \underline{\mathrm{C}}\right), 26.9\left(\underline{\mathrm{Me}}_{3} \mathrm{C}\right), 26.7\left(\mathrm{Me}_{2} \mathrm{C}\right) . \mathrm{MS}$ (EI) $m / z(\%): 315\left(\mathbf{M}^{+}-15,66\right)$.

(5S)-5-[(1S)-1-Hydroxy-2-pivaloyloxyethyl]-2(5H)-furanone (5e). A solution of alcohol 8 (5.06 g, $20.5 \mathrm{mmol})$ in dry $\mathrm{CH}_{2} \mathrm{Cl}_{2}(20 \mathrm{~mL})$ was added dropwise to a stirred solution of oxalyl chloride $(1.9 \mathrm{~mL}, 21.8 \mathrm{mmol})$ and DMSO $(3.1 \mathrm{~mL}, 43.7 \mathrm{mmol})$ in dry $\mathrm{CH}_{2} \mathrm{Cl}_{2}(60 \mathrm{~mL})$ at $-78{ }^{\circ} \mathrm{C}$ under a nitrogen atmosphere. The mixture was stirred for $30 \mathrm{~min}$, triethylamine $(12 \mathrm{~mL})$ was added dropwise and the solution was allowed to reach room temperature. Then, the solvent was evaporated, the resulting residue was dissolved in $\mathrm{CH}_{2} \mathrm{Cl}_{2}(75 \mathrm{~mL})$ and the solution was washed with brine $(2 \times 30 \mathrm{~mL})$. The organic fraction was dried $\left(\mathrm{MgSO}_{4}\right)$ and the solvent evaporated to give a residue that was used in the next step without further purification. (Methoxycarbonylmethylene)triphenylphosphorane $(7.22 \mathrm{~g}, 21.6 \mathrm{mmol})$ was added in small portions to a gently stirred solution of this oily residue in methanol $(50 \mathrm{~mL})$, and the mixture was stirred at room temperature overnight. The solvent was removed and the resulting solid residue was extracted $\left(\mathrm{Et}_{2} \mathrm{O}, 2 \times 50 \mathrm{~mL}\right)$. The ether was removed under vacuum and the residue was dissolved in methanol $(50 \mathrm{~mL})$ and cooled to $0{ }^{\circ} \mathrm{C}$. While vigorously stirring, concd. $\mathrm{HCl}$ was slowly added $(16.6 \mathrm{~mL})$ and stirring was continued for $30 \mathrm{~min}$ at room temperature. The solvent was evaporated and the residue was purified by FCC (hexanes/EtOAc, 1:1) to afford the title compound 5e (2.4 g, 51\%) as a colorless solid and the $(E)-\alpha, \beta$-unsaturated ester 9 (395 mg, 7\%) as a colorless oil.

5e. $\mathrm{Mp} 88-91{ }^{\circ} \mathrm{C}$ (hexanes/EtOAc). $[\alpha]_{\mathrm{D}}-66.5$ (c 2.6, $\mathrm{CHCl}_{3}$ ); IR (ATR): 3428, 2973, 1746, $1696 \mathrm{~cm}^{-1} .{ }^{1} \mathrm{H}$ NMR $\left(250 \mathrm{MHz}, \mathrm{CDCl}_{3}\right) \delta_{\mathrm{H}} 7.52\left(\mathrm{dd}, 1 \mathrm{H}, J_{4,3} 5.8 \mathrm{~Hz}, J_{4,5} 4.5 \mathrm{~Hz}, \mathrm{H}-4\right), 6.25(\mathrm{dd}$, $\left.1 \mathrm{H}, J_{3,4} 5.8 \mathrm{~Hz}, J_{3,5} 2.2 \mathrm{~Hz}, \mathrm{H}-3\right), 5.16-5.10(\mathrm{~m}, 1 \mathrm{H}, \mathrm{H}-5), 4.32$ (dd, $1 \mathrm{H}, J 10.8,6.1 \mathrm{~Hz}, \mathrm{CH}_{2} \mathrm{O}$ ), 4.24 (dd, 1H, J 10.8, $4.5 \mathrm{~Hz}, \mathrm{CH}_{2} \mathrm{O}$ ), 4.17-4.05 (m, 1H, CHO), 2.64 (d, 1H, J 5.9 Hz, OH), 1.25 $\left(\mathrm{s}, 9 \mathrm{H}, t\right.$-Bu). ${ }^{13} \mathrm{C}$ NMR $\left(62.5 \mathrm{MHz}, \mathrm{CDCl}_{3}\right): 178.5(\mathrm{C}=\mathrm{O}), 172.0(\mathrm{C}=\mathrm{O}), 152.7(\mathrm{C}-4), 122.8(\mathrm{C}-$ 3), 82.9 (C-5), 69.8 (CHO), $64.6\left(\mathrm{CH}_{2} \mathrm{O}\right), 38.6\left(\mathrm{Me}_{3} \mathrm{C}\right), 26.9$ ( $\left.\mathrm{Me}_{3} \mathrm{C}\right) . \mathrm{MS}(\mathrm{EI}) \mathrm{m} / z(\%): 251.0$ $\left(\mathrm{M}+\mathrm{Na}^{+}\right.$, 93), $267.0\left(\mathrm{M}+\mathrm{K}^{+}\right.$, 100). Anal. Calcd. for $\mathrm{C}_{11} \mathrm{H}_{16} \mathrm{O}_{5}: \mathrm{C}, 57.88 ; \mathrm{H}, 7.07$. Found: $\mathrm{C}$, $57.52 ; \mathrm{H}, 7.06 \%$.

9. $[\alpha]_{\mathrm{D}}+465.0\left(c\right.$ 0.4, $\mathrm{CHCl}_{3}$ ). IR (ATR): $3437,2960,2909,2874,1705,1660 \mathrm{~cm}^{-1} .{ }^{1} \mathrm{H} \mathrm{NMR}$ $\left(250 \mathrm{MHz}, \mathrm{CDCl}_{3}\right) \delta_{\mathrm{H}} 6.98\left(\mathrm{dd}, J_{3,2} 15.7 \mathrm{~Hz}, J_{3,4} 4.7 \mathrm{~Hz}, 1 \mathrm{H}, \mathrm{H}-3\right), 6.19\left(\mathrm{dd}, J_{2,3} 15.7 \mathrm{~Hz}, J_{2,4} 1.8\right.$ Hz, 1H, H-2), 4.35-4.22 (m, 2H, H-4, H-6), 4.17 (dd, J Jem 11.8 Hz, J6,5 5.8 Hz, 1H, H-6), 3.90$3.79(\mathrm{~m}, 1 \mathrm{H}, \mathrm{H}-5), 3.76\left(\mathrm{~s}, 3 \mathrm{H}, \mathrm{CO}_{2} \mathrm{Me}\right), 3.05(\mathrm{~s}, 2 \mathrm{H}, 2 \mathrm{OH}), 1.23(\mathrm{~s}, 9 \mathrm{H}, t-\mathrm{Bu}) .{ }^{13} \mathrm{C} \mathrm{NMR}(62.5$ $\left.\mathrm{MHz}, \mathrm{CDCl}_{3}\right) \delta_{\mathrm{C}} 179.5(\mathrm{C}=\mathrm{O}), 167.0(\mathrm{C}=\mathrm{O}), 146.5(\mathrm{C}-3), 122.8(\mathrm{C}-2), 72.5(\mathrm{C}-5), 71.3(\mathrm{C}-4)$, 65.4 (C-6), $52.1\left(\mathrm{CO}_{2} \underline{\mathrm{Me}}\right), 39.3\left(\mathrm{Me}_{3} \underline{\mathrm{C}}\right), 27.5\left(\underline{\mathrm{Me}}_{3} \mathrm{C}\right) . \mathrm{MS}(\mathrm{ESI}) \mathrm{m} / z(\%): 283.1\left(\mathrm{M}^{\mathrm{N}} \mathrm{Na}^{+}, 10\right)$, 
$299.1\left(\mathrm{M}+\mathrm{K}^{+}, 63\right), 543.0\left(2 \mathrm{M}+\mathrm{Na}^{+}, 100\right)$. Anal. Calcd. for $\mathrm{C}_{12} \mathrm{H}_{20} \mathrm{O}_{6} \cdot 1 / 2 \mathrm{H}_{2} \mathrm{O}: \mathrm{C}, 53.52 ; \mathrm{H}, 7.86$. Found: C, 53.10; H, 7.72\%.

(5S)-5-[(1S)-1,2-Dipivaloyloxyethyl]-2(5H)-furanone (5f). Pivaloyl chloride (0.8 $\mathrm{mL}, 6.50$ mmol) was added dropwise to an ice-cooled solution of the furanone 5e (492 $\mathrm{mg}, 2.16 \mathrm{mmol}$ ), DMAP (54 mg, $0.44 \mathrm{mmol})$ and dry pyridine $(1 \mathrm{~mL})$ in dry $\mathrm{CH}_{2} \mathrm{Cl}_{2}(10 \mathrm{~mL})$ under a nitrogen atmosphere. The mixture was stirred overnight as it came to room temperature. Then, $\mathrm{CH}_{2} \mathrm{Cl}_{2}$ $(20 \mathrm{~mL})$ was added and the solution washed with brine $(2 \times 20 \mathrm{~mL})$. The organic layer was dried $\left(\mathrm{MgSO}_{4}\right)$, the solvent evaporated and the residue was purified by FCC (EtOAc/hexanes, 1:3) to give the title compound $\mathbf{5 f}$ as a colorless solid $(517 \mathrm{mg}, 77 \%)$ : $\mathrm{mp} 94-94{ }^{\circ} \mathrm{C}\left(\mathrm{Et}_{2} \mathrm{O} /\right.$ hexanes $) .[\alpha]_{\mathrm{D}}$ -147.3 ( c 1.1, $\mathrm{CHCl}_{3}$ ); IR (ATR): 3113, 2968, 1730, $1757 \mathrm{~cm}^{-1} .{ }^{1} \mathrm{H}$ NMR $\left(250 \mathrm{MHz}, \mathrm{CDCl}_{3}\right) \delta_{\mathrm{H}}$ $7.40\left(\mathrm{dd}, J_{4,3} 5.8 \mathrm{~Hz}, J_{4,5} 1.6 \mathrm{~Hz}, 1 \mathrm{H}, \mathrm{H}-4\right), 6.18\left(\mathrm{dd}, J_{3,4} 5.8 \mathrm{~Hz}, J_{3,5} 2.2 \mathrm{~Hz}, 1 \mathrm{H}, \mathrm{H}-3\right), 5.36$ (ddd, $\left.J_{6,7} 7.4 \mathrm{~Hz}, J 4.8,2.5 \mathrm{~Hz}, 1 \mathrm{H}, \mathrm{CHO}\right), 5.25$ (dd, $\left.J 2.5 \mathrm{~Hz}, J_{5,4} 1.6 \mathrm{~Hz}, 1 \mathrm{H}, \mathrm{H}-5\right), 4.48$ (dd, $J 11.8$, $4.8 \mathrm{~Hz}, 1 \mathrm{H}, \mathrm{CH}_{2} \mathrm{O}$ ), 4.31 (dd, $J 11.8,7.4 \mathrm{~Hz}, 1 \mathrm{H}, \mathrm{CH}_{2} \mathrm{O}$ ), $1.22(\mathrm{~s}, 9 \mathrm{H}, t-\mathrm{Bu}), 1.67$ (s, 9H, $\left.t-\mathrm{Bu}\right)$. ${ }^{13} \mathrm{C} \mathrm{NMR}\left(62.5 \mathrm{MHz}, \mathrm{CDCl}_{3}\right) \delta_{\mathrm{C}} 177.1(\mathrm{C}=\mathrm{O}), 151.7(\mathrm{C}-4), 122.7(\mathrm{C}-3), 81.1$ (C-5), 68.1 (CHO), $62.3\left(\mathrm{CH}_{2} \mathrm{O}\right), 38.6\left(\mathrm{Me}_{3} \underline{\mathrm{C}}\right), 38.5\left(\mathrm{Me}_{3} \underline{\mathrm{C}}\right), 26.8\left(\underline{\mathrm{Me}}_{3} \mathrm{C}\right), 26.7\left(\underline{\mathrm{Me}}_{3} \mathrm{C}\right) . \mathrm{MS}(\mathrm{ESI}) \mathrm{m} / z(\%)$ : 335.1 (M+Na $\left.{ }^{+}, 19\right), 351.0\left(\mathrm{M}+\mathrm{K}^{+}, 100\right)$. Anal. Calcd. for $\mathrm{C}_{16} \mathrm{H}_{24} \mathrm{O}_{6}: \mathrm{C}, 61.52 ; \mathrm{H}, 7.74$. Found: $\mathrm{C}$, 61.34; H, 7.58\%.

(5S)-5-[(1S)-1-Isovaleroyloxy-2-pivaloyloxyethyl]-2(5H)-furanone (5g). Isovaleroyl chloride $(360 \mu \mathrm{L}, 2.95 \mathrm{mmol})$ was added to an ice-cooled solution of the furanone 5e (336 mg, 1.47 mmol) and dry pyridine $(0.5 \mathrm{~mL})$ in dry $\mathrm{CH}_{2} \mathrm{Cl}_{2}(7 \mathrm{~mL})$ under a nitrogen atmosphere. The mixture was stirred overnight as it came to room temperature. Then, $\mathrm{CH}_{2} \mathrm{Cl}_{2}(20 \mathrm{~mL})$ was added and the solution washed with brine $(2 \times 20 \mathrm{~mL})$. The organic layer was dried $\left(\mathrm{MgSO}_{4}\right)$, the solvent evaporated and the residue was purified by crystallization $\left(\mathrm{Et}_{2} \mathrm{O} /\right.$ hexanes) to give the title compound $\mathbf{5 g}$ as a colorless solid (354 mg, 77\%), which slowly decomposes: $\mathrm{mp} 71-72{ }^{\circ} \mathrm{C}$ (Et ${ }_{2} \mathrm{O} /$ hexanes). IR (ATR): 3095, 2967, 2936, 2872, 1722, $1764 \mathrm{~cm}^{-1} .{ }^{1} \mathrm{H}$ NMR $(250 \mathrm{MHz}$, $\left.\mathrm{CDCl}_{3}\right) \delta_{\mathrm{H}} 7.42\left(\mathrm{dd}, J_{4,3} 5.7 \mathrm{~Hz}, J_{4,5} 1.7 \mathrm{~Hz}, 1 \mathrm{H}, \mathrm{H}-4\right), 6.20\left(\mathrm{dd}, J_{3,4} 5.7 \mathrm{~Hz}, J_{3,5} 2.0 \mathrm{~Hz}, 1 \mathrm{H}, \mathrm{H}-3\right)$, 5.42-5.37 (m, 1H, CHO), 5.28-5.21 (m, 1H, H-5), 4.45 (dd, J 11.7, $4.8 \mathrm{~Hz}, 1 \mathrm{H}, \mathrm{CH}_{2} \mathrm{O}$ ), 4.29 (dd, $J$ 11.7, $\left.6.9 \mathrm{~Hz}, 1 \mathrm{H}, \mathrm{CH}_{2} \mathrm{O}\right), 2.20-2.14\left(\mathrm{~m}, 2 \mathrm{H}, \mathrm{COCH}_{2} \mathrm{CHMe}_{2}\right), 2.15-1.90(\mathrm{~m}, 1 \mathrm{H}$, $\mathrm{COCH}_{2} \underline{\mathrm{HMe}}_{2}$ ), 1.22 (s, 9H, $t$-Bu), 0.95 (dd, $\left.6 \mathrm{H}, J 6.5,0.8 \mathrm{~Hz}, \mathrm{COCH}_{2} \mathrm{CHMe}_{2}\right) .{ }^{13} \mathrm{C} \mathrm{NMR}(62.5$ $\left.\mathrm{MHz}, \mathrm{CDCl}_{3}\right) \delta_{\mathrm{C}} 177.5(\mathrm{C}=\mathrm{O}), 171.7(\mathrm{C}=\mathrm{O}), 171.6(\mathrm{C}=\mathrm{O}) ; 151.7(\mathrm{C}-4), 122.9(\mathrm{C}-3), 81.0(\mathrm{C}-5)$, 68.4 (CHO), $62.2 \quad\left(\mathrm{CH}_{2} \mathrm{O}\right), 42.7 \quad\left(\mathrm{COCH}_{2} \mathrm{CHMe}_{2}\right), \quad 38.5 \quad\left(\mathrm{Me}_{3} \underline{\mathrm{C}}\right), 26.8 \quad\left(\underline{\mathrm{Me}}_{3} \mathrm{C}\right), 25.2$ $\left(\mathrm{COCH}_{2} \underline{\mathrm{CHMe}}{ }_{2}\right), 22.0\left(\mathrm{COCH}_{2} \mathrm{CHMe}_{2}\right)$. MS (ESI) $\mathrm{m} / \mathrm{z}(\%)$ : $335.1\left(\mathrm{M}+\mathrm{Na}^{+}, 100\right), 351.1\left(\mathrm{M}+\mathrm{K}^{+}\right.$, 5). HRMS (FAB+): calculated for $\left[\mathrm{C}_{16} \mathrm{H}_{24} \mathrm{O}_{6}+\mathrm{H}\right]^{+} 313.1651$; found 313.1653 .

(3aS,4S,6aR)-4-[(1S)-1,2-Dipivaloyloxyethyl]-3a,4,6,6a-tetrahydro-3H-furo[3,4-c]pyrazol-6one (10). To an ice-cooled stirred solution of the furanone $\mathbf{5 f}(380 \mathrm{mg}, 1.22 \mathrm{mmol})$ in THF (5 $\mathrm{mL}$ ) was added an ethereal solution of diazomethane $(\mathrm{ca} .4 .8 \mathrm{mmol})$ prepared from $\mathrm{N}$-methyl- $\mathrm{N}$ nitroso-4-toluensulfonamide $(1.04 \mathrm{~g}, 4.84 \mathrm{mmol})$. The mixture was stirred at room temperature, protected from light, for $48 \mathrm{~h}$. Removal of the solvent afforded the title compound $\mathbf{1 0}$ as a

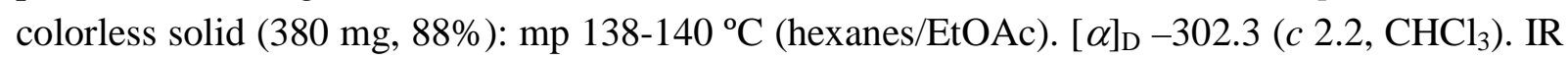
(ATR): 2980, 2928, 2907, 2871, 1769, $1726 \mathrm{~cm}^{-1} .{ }^{1} \mathrm{H}$ NMR $\left(250 \mathrm{MHz}, \mathrm{CDCl}_{3}\right) \delta_{\mathrm{H}} 5.56$ (ddd, 
$\left.J_{6 \mathrm{a}, 3 \mathrm{a}} 9.3 \mathrm{~Hz}, J_{6 \mathrm{a}, 3} 2.5 \mathrm{~Hz}, J_{6 \mathrm{a}, 3} 1.4 \mathrm{~Hz}, 1 \mathrm{H}, \mathrm{H}-6 \mathrm{a}\right), 5.27$ (ddd, J 7.0, 4.3, 2.4 Hz, 1H, CHO), 4.97 (ddd, $\left.J_{\text {gem }} 11.8 \mathrm{~Hz}, J_{3,3 \mathrm{a}} 9.0 \mathrm{~Hz}, J_{3,6 \mathrm{a}} 1.4 \mathrm{~Hz}, 1 \mathrm{H}, \mathrm{H}-3\right), 4.81$ (ddd, $J_{\text {gem }} 11.8 \mathrm{~Hz}, J_{3,3 \mathrm{a}} 3.6 \mathrm{~Hz}, J_{3,6 \mathrm{a}}$ $2.5 \mathrm{~Hz}, 1 \mathrm{H}, \mathrm{H}-3), 4.38\left(\mathrm{dd}, J 11.9,4.3 \mathrm{~Hz}, 1 \mathrm{H}, \mathrm{CH}_{2} \mathrm{O}\right), 4.30\left(\mathrm{dd}, J_{4,3 \mathrm{a}} 3.2 \mathrm{~Hz}, J_{4,7} 2.4 \mathrm{~Hz}, 1 \mathrm{H}, \mathrm{H}-\right.$ 4), 4.14 (dd, $J 11.9,7.0 \mathrm{~Hz}, 1 \mathrm{H}, \mathrm{CH}_{2} \mathrm{O}$ ), 2.7 (dddd, $J_{3 \mathrm{a}, 6 \mathrm{a}} 9.3 \mathrm{~Hz}, J_{3 \mathrm{a}, 3} 9.0 \mathrm{~Hz}, J_{3 \mathrm{a}, 3} 3.6 \mathrm{~Hz}, J_{3 \mathrm{a}, 4} 3.2$ $\mathrm{Hz}, 1 \mathrm{H}, \mathrm{H}-3 \mathrm{a}), 1.24\left(\mathrm{~s}, 9 \mathrm{H}, t\right.$-Bu), $1.18\left(\mathrm{~s}, 9 \mathrm{H}, t\right.$-Bu). ${ }^{13} \mathrm{C} \mathrm{NMR}\left(62.5 \mathrm{MHz}, \mathrm{CDCl}_{3}\right) \delta_{\mathrm{C}} 178.3$ $(\mathrm{C}=\mathrm{O}), 177.8(\mathrm{C}=\mathrm{O}), 167.3(\mathrm{C}=\mathrm{O}), 93.7(\mathrm{C}-4), 86.6(\mathrm{C}-8), 83.6(\mathrm{C}-6 \mathrm{a}), 71.7(\mathrm{C}-7), 62.8(\mathrm{C}-3)$, $39.5\left(\mathrm{Me}_{3} \underline{\mathrm{C}}\right), 39.2\left(\mathrm{Me}_{3} \underline{\mathrm{C}}\right), 34.2$ (C-3a), $27.5\left(\mathrm{Me}_{3} \mathrm{C}\right)$. MS (ESI) $\mathrm{m} / z(\%): 377.2\left(\mathrm{M}^{2} \mathrm{Na}^{+}, 100\right)$. Anal. Calcd. for $\mathrm{C}_{17} \mathrm{H}_{26} \mathrm{~N}_{2} \mathrm{O}_{6}: \mathrm{C}, 57.61 ; \mathrm{H}, 7.39 ; \mathrm{N}, 7.90$. Found: C, 58.01; H, 7.64; N, 7.73\%.

(5S)-4-Methyl-5-[(1S)-1,2-dipivaloyloxyethyl]-2(5H)-furanone $(5 \mathrm{~h})$. A solution of the pyrazolone $10(360 \mathrm{mg}, 1.02 \mathrm{mmol})$ in dioxane $(20 \mathrm{~mL})$ was heated at reflux for $48 \mathrm{~h}$. The solvent was evaporated and the residue was purified by FCC (from hexanes to hexanes/EtOAc 4:1) to afford the title compound $\mathbf{5 h}$ as a colorless solid (203 $\mathrm{mg}, 61 \%)$ : $\mathrm{mp} 89-91{ }^{\circ} \mathrm{C}$ (hexanes/EtOAc): $[\alpha]_{\mathrm{D}}-85.0$ ( $c$ 1.6 $\mathrm{CHCl}_{3}$ ). IR (ATR): 2964, 2924, 2853, 1768, 1736, 1722, $1479 \mathrm{~cm}^{-1} .{ }^{1} \mathrm{H}$ NMR $\left(250 \mathrm{MHz}, \mathrm{CDCl}_{3}\right) \delta_{\mathrm{H}} 5.92-5.82(\mathrm{~m}, 1 \mathrm{H}, \mathrm{H}-3), 5.48$ (ddd, J 8.1, 4.5, $1.5 \mathrm{~Hz}$, 1H, CHO), 4.97 (broad s, 1H, H-5), 4.46 (dd, J 11.6, $4.5 \mathrm{~Hz}, 1 \mathrm{H}, \mathrm{CH}_{2} \mathrm{O}$ ), 4.27 (dd, J 11.6, 8.1 $\mathrm{Hz}, 1 \mathrm{H}, \mathrm{CH}_{2} \mathrm{O}$ ), 2.08 (br s, 3H, Me), 1.19 (s, 9H, $t$-Bu), 1.14 (s, 9H, $t$-Bu). ${ }^{13} \mathrm{C} \mathrm{NMR}(62.5 \mathrm{MHz}$, $\left.\mathrm{CDCl}_{3}\right) \delta_{\mathrm{C}} 178.3(\mathrm{C}=\mathrm{O}), 177.7(\mathrm{C}=\mathrm{O}), 172.5(\mathrm{C}=\mathrm{O}), 165.0(\mathrm{C}-4), 119.1(\mathrm{C}-3), 83.0(\mathrm{C}-5), 67.3$ (CHO), $63.6\left(\mathrm{CH}_{2} \mathrm{O}\right), 39.4\left(\mathrm{Me}_{3} \underline{\mathrm{C}}\right), 39.2\left(\mathrm{Me}_{3} \underline{\mathrm{C}}\right), 27.5\left(\mathrm{Me}_{3} \mathrm{C}\right), 14.2(\mathrm{Me}) . \mathrm{MS}(\mathrm{EI}) \mathrm{m} / \mathrm{z}(\%)$ : $349.2\left(\mathrm{M}+\mathrm{Na}^{+}, 100\right)$. HRMS (FAB+): calculated for $\left[\mathrm{C}_{17} \mathrm{H}_{26} \mathrm{O}_{6}+\mathrm{H}\right]^{+} 349.1622$; found, 349.1612.

General procedure for the photocycloadditions of $2(5 \mathrm{H})$-furanones to alkenes. Irradiations were performed in a conventional photochemical reactor (two-necked vessel fitted with a Pyrex or quartz immersion-type cooling jacket) using a medium-pressure, $125 \mathrm{~W}$ mercury lamp. Methanol at $-15^{\circ} \mathrm{C}$ was used for the refrigeration of the immersion well jacket. The vessel was externally cooled at $-20{ }^{\circ} \mathrm{C}$ with a dry ice/ $\mathrm{CCl}_{4}$ bath. The progress of the reaction was monitored by $\mathrm{GC}$ analysis of aliquot samples. For the reactions with ethylene, this gas was bubbled through the solution for $15 \mathrm{~min}$ before turning the lamp on and a slow flow of ethylene was maintained throughout the irradiation.

$(1 R, 4 S, 5 S)$-, 11a, and $(1 S, 4 S, 5 R)-4-[(1 S)$-2-Benzyloxy-1-hydroxyethyl]-3-oxabicyclo[3.2.0]heptan-2-one (12a). Following the general procedure, a solution of the furanone $\mathbf{5 a}(573 \mathrm{mg}$, $2.45 \mathrm{mmol})$ in acetone $(300 \mathrm{~mL})$ saturated with ethylene was irradiated through a Pyrex filter for $2 \mathrm{~h} 10 \mathrm{~min}$. Evaporation of the solvent and purification by FCC (hexanes/EtOAc, 4:1) afforded a 89:11 mixture of cycloadducts 11a and 12a (499 mg, 78\%). Repeated FCC (from hexanes to hexanes/EtOAc, 4:1) provided a pure sample of isomer 11a as a colorless oil and enriched fractions of the title compound $\mathbf{1 2 \mathbf { a }}$ also as a colorless oil.

11a + 12a. MS (ESI) $m / z(\%): 263.1\left(\mathrm{M}+\mathrm{H}^{+}, 3\right), 285.1\left(\mathrm{M}+\mathrm{Na}^{+}, 17\right), 301.1\left(\mathrm{M}+\mathrm{K}^{+}, 100\right)$. Anal. Calcd. for $\mathrm{C}_{15} \mathrm{H}_{18} \mathrm{O}_{4}$ : C, 68.68; H, 6.92. Found: C, 68.29; H, 6.97\%.

11a. $[\alpha]_{\mathrm{D}}+15.7\left(c\right.$ 4.4, $\left.\mathrm{CHCl}_{3}\right)$. IR (ATR): 3413, 2942, 2864, $1744 \mathrm{~cm}^{-1} .{ }^{1} \mathrm{H} \mathrm{NMR}(250 \mathrm{MHz}$, $\left.\mathrm{CDCl}_{3}\right) \delta_{\mathrm{H}} 7.45-7.25(\mathrm{~m}, 5 \mathrm{H}, \mathrm{H}-\mathrm{Ar}), 4.57\left(\mathrm{~s}, 2 \mathrm{H}, \mathrm{CH}_{2} \mathrm{Ph}\right), 4.38\left(\mathrm{~d}, J_{4,5} 2.3 \mathrm{~Hz}, 1 \mathrm{H}, \mathrm{H}-4\right), 3.88-$ $3.78(\mathrm{~m}, 1 \mathrm{H}, \mathrm{CHO}), 3.61\left(\mathrm{~s}, 1 \mathrm{H}, \mathrm{CH}_{2} \mathrm{O}\right), 3.89\left(\mathrm{~s}, 1 \mathrm{H}, \mathrm{CH}_{2} \mathrm{O}\right), 3.25-3.08(\mathrm{~m}, 2 \mathrm{H}, 2 \mathrm{H}-$ 
cyclobutane), 2.66-2.31 (m, 3H, 2H-cyclobutane, $\mathrm{OH}), 2.24-2.03\left(\mathrm{~m}, 2 \mathrm{H}, 2 \mathrm{H}\right.$-cyclobutane). ${ }^{13} \mathrm{C}$ NMR (62.5 MHz, $\left.\mathrm{CDCl}_{3}\right) \delta_{\mathrm{C}} 180.3(\mathrm{C}=\mathrm{O}), 137.3,128.3,127.7,127.6(\mathrm{C}-\mathrm{Ar}), 84.4(\mathrm{C}-4), 73.4$ $\left(\underline{\mathrm{CH}}_{2} \mathrm{Ph}\right), 72.0(\mathrm{CHO}), 70.5\left(\mathrm{CH}_{2} \mathrm{O}\right), 38.8,36.9$ (C-1/C-5), 24.6, 23.5 (C-6/C-7).

12a. (data extracted from an enriched sample) IR (ATR): 3434, 2951, 2868, 1756, $1721 \mathrm{~cm}^{-1} .{ }^{1} \mathrm{H}$ NMR $\left(250 \mathrm{MHz}, \mathrm{CDCl}_{3}\right) \delta_{\mathrm{H}}$ 7.55-7.23 (m, 5H, H-Ar), 4.60 (d, $\left.J_{\text {gem }} 11.8 \mathrm{~Hz}, 1 \mathrm{H}, \mathrm{C}_{2} \mathrm{Ph}\right), 4.52$ $\left(\mathrm{d}, J_{\text {gem }} 11.8 \mathrm{~Hz}, 1 \mathrm{H}, \mathrm{C}_{2} \mathrm{Ph}\right.$ ), 4.47 (dd, J 7.7, $\left.5.2 \mathrm{~Hz}, 1 \mathrm{H}, \mathrm{H}-4\right), 4.07$ (dt $J 7.2,5.1 \mathrm{~Hz}, 1 \mathrm{H}$, CHO), 3.52 (dd, $J$ 10.0, $3.9 \mathrm{~Hz}, 1 \mathrm{H}, \mathrm{CH}_{2} \mathrm{O}$ ), 3.49 (dd, $J$ 10.0, $5.0 \mathrm{~Hz}, 1 \mathrm{H}, \mathrm{CH}_{2} \mathrm{O}$ ), 3.20-3.05 (m, 2H, H-1, H-5), 2.60-1.95 (m, 4H, 2H-6, 2H-7), 1.90-1.60 (m, 1H, OH). ${ }^{13} \mathrm{C}$ NMR (62.5 MHz, $\left.\mathrm{CDCl}_{3}\right) \delta_{\mathrm{C}} 179.4(\mathrm{C}=\mathrm{O}), 137.3,129.4,128.2,127.5(\mathrm{C}-\mathrm{Ar}), 83.0(\mathrm{C}-4), 73.5\left(\mathrm{CH}_{2} \mathrm{O}\right), 70.3$ (CHO), $69.5\left(\underline{\mathrm{CH}}_{2} \mathrm{Ph}\right), 40.0,36.2$ (C-1/C-5), 23.0, 19.8 (C-6/C-7).

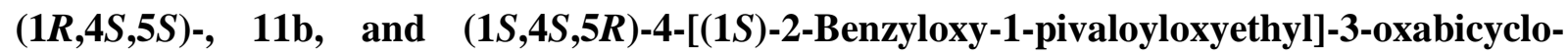
[3.2.0]heptane-2-one (12b). Following the general procedure, a solution of the furanone $\mathbf{5 b}$ (119 $\mathrm{mg}, 0.37 \mathrm{mmol})$ in acetone $(65 \mathrm{~mL})$ saturated with ethylene was irradiated through a Pyrex filter for $5 \mathrm{~h} 20 \mathrm{~min}$. Evaporation of the solvent and purification by FCC (hexanes/EtOAc, 1:4) afforded a 90:10 mixture of cycloadducts $\mathbf{1 1 b}$ and 12b (96 mg, 74\%). Repeated FCC (hexanes/EtOAc, 9:1) provided a pure sample of isomer 11b and enriched fractions of the title compound $\mathbf{1 2 b}$ as colorless oils.

11b + 12b. MS (ESI) $\mathrm{m} / z(\%): 342\left(\mathrm{M}+\mathrm{H}^{+}\right), 36922\left(\mathrm{M}+\mathrm{Na}^{+}, 38\right), 385.1\left(\mathrm{M}+\mathrm{K}^{+}, 100\right)$. Anal. Calcd. for $\mathrm{C}_{20} \mathrm{H}_{26} \mathrm{O}_{5}$ : C, 69.34; H, 7.56. Found: C, 69.34; H, 7.65\%.

11b. $[\alpha]_{\mathrm{D}}+68.2\left(c\right.$ 1.1, $\left.\mathrm{CHCl}_{3}\right)$; IR (ATR): 2959, 2870, 1771, $1728 \mathrm{~cm}^{-1} .{ }^{1} \mathrm{H}$ NMR $(250 \mathrm{MHz}$, $\left.\mathrm{CDCl}_{3}\right) \delta_{\mathrm{H}} 7.40-7.26(\mathrm{~m}, 5 \mathrm{H}, \mathrm{H}-\mathrm{Ar}), 5.09$ (ddd, $\left.J 7.0,6.2,2.0 \mathrm{~Hz}, 1 \mathrm{H}, \mathrm{CHO}\right), 4.70(\mathrm{dd}, J 2.0,1.1$ $\mathrm{Hz}, 1 \mathrm{H}, \mathrm{H}-4), 4.55$ (s, 2H, $\mathrm{CH}_{2} \mathrm{O}$ ), 3.69-3.52 (m, 2H, $\left.\underline{\mathrm{C}}_{2} \mathrm{Ph}\right), 3.10-2.88$ (m, 2H, H-1, H-5), $2.60-$ $2.45(\mathrm{~m}, 1 \mathrm{H}, \mathrm{H}$-cyclobutane $), 2.45-2.30(\mathrm{~m}, 1 \mathrm{H}, \mathrm{H}$-cyclobutane $), 2.22-2.05(\mathrm{~m}, 2 \mathrm{H}, 2 \mathrm{H}-$ cyclobutane), $1.19\left(\mathrm{~s}, 9 \mathrm{H}, t\right.$-Bu). ${ }^{13} \mathrm{C}$ NMR $\left(62.5 \mathrm{MHz}, \mathrm{CDCl}_{3}\right) \delta_{\mathrm{C}} 180.5(\mathrm{C}=\mathrm{O}), 178.0(\mathrm{C}=\mathrm{O})$, 138.0, 128.9, 128.1 (C-Ar), 83.7 (C-4), $73.9\left(\underline{\mathrm{CH}}_{2} \mathrm{Ph}\right), 73.1(\mathrm{CHO}), 68.1\left(\mathrm{CH}_{2} \mathrm{O}\right), 39.4\left(\underline{\mathrm{CMe}}_{3}\right)$, 39.1 (C-1/C-5), 36.91 (C-1/C-5), 27.5 ( $\mathrm{CH}_{3}, \mathrm{CMe}_{3}$ ), 25.4 (C-6/C-7), 24.2 (C-6/C-7).

12b. (data extracted from an enriched sample) IR (ATR): 2960, 2924, 2853, 1772, $1726 \mathrm{~cm}^{-1} .{ }^{1} \mathrm{H}$ NMR $\left(250 \mathrm{MHz}, \mathrm{CDCl}_{3}\right) \delta_{\mathrm{H}}$ 7.45-7.27 (m, 5H, H-Ar), 5.30-5.20 (m, 1H, CHO), 4.72 (dd, J 8.6, $5.3 \mathrm{~Hz}, 1 \mathrm{H}, \mathrm{H}-4), 4.62-4.57\left(\mathrm{C}_{2} \mathrm{Ph}\right), 3.59$ (dd, $\left.J 11.1,3.7 \mathrm{~Hz}, 1 \mathrm{H}, \mathrm{CH}_{2} \mathrm{O}\right), 3.48$ (dd, $J 11.1,3.7$ $\mathrm{Hz}, 1 \mathrm{H}, \mathrm{CH}_{2} \mathrm{O}$ ), 3.29-2.98 (m, 2H, H-1, H-5), 2.64-1.96 (m, 4H, 4H-cyclobutane), 1.27 (s, 9H, $t$ $\mathrm{Bu}) .{ }^{13} \mathrm{C}$ NMR $\left(62.5 \mathrm{MHz}, \mathrm{CDCl}_{3}\right) \delta_{\mathrm{C}} 177.5(\mathrm{C}=\mathrm{O}), 177.2(\mathrm{C}=\mathrm{O}), 137.2,129.4,128.6,127.4(\mathrm{C}-$ Ar), 83.0 (C-4), $73.4\left(\underline{\mathrm{CH}}_{2} \mathrm{Ph}\right), 71.6(\mathrm{CHO}), 67.9\left(\mathrm{CH}_{2} \mathrm{O}\right), 39.9(\mathrm{C}-1), 38.6\left(\mathrm{Me}_{3} \underline{\mathrm{C}}\right), 36.2(\mathrm{C}-5)$, $26.7\left(\underline{\mathrm{Me}}_{3} \mathrm{C}\right), 24.7,23.5$ (C-6/C-7).

$(1 R, 4 S, 5 S)$-, 11c, and $(1 S, 4 S, 5 R)-4-[(1 S)$-1-Benzoyloxy-2-benzyloxyethyl]-3-oxabicyclo[3.2.0]heptan-2-one (12c). Following the general procedure, a solution of the furanone 5c (150 $\mathrm{mg}, 0.443 \mathrm{mmol})$ in acetone $(65 \mathrm{~mL})$ saturated with ethylene was irradiated through a Pyrex filter for $1 \mathrm{~h} 35 \mathrm{~min}$. Evaporation of the solvent and purification by FCC (hexanes/EtOAc, 4:1) furnished a 80:20 mixture of cycloadducts 11c and 12c (114 mg, 70\%). Repeated FCC (hexanes/EtOAc, 9:1) provided a pure sample of isomer 11c and enriched fractions of 12c as colorless oils. 
11c + 12c. MS (ESI) $\mathrm{m} / \mathrm{z}(\%): 389.2\left(\mathrm{M}+\mathrm{Na}^{+}, 34\right), 405\left(\mathrm{M}+\mathrm{K}^{+}, 100\right)$. HRMS (FAB+): calculated for $\left[\mathrm{C}_{20} \mathrm{H}_{22} \mathrm{O}_{5}+\mathrm{H}\right]^{+}$367.1545; found, 367.1543.

11c: $[\alpha]_{\mathrm{D}}+7.4\left(c\right.$ 0.4, $\left.\mathrm{CHCl}_{3}\right)$. IR (ATR): 3062, 3030, 2947, 2865, 1769, $1719 \mathrm{~cm}^{-1} .{ }^{1} \mathrm{H}$ NMR $\left(250 \mathrm{MHz}, \mathrm{CDCl}_{3}\right) \delta_{\mathrm{H}} 7.97$ (d, J 7.5 Hz, 2H, H-Ar), 7.60 (t, J 7.5 Hz, 1H, H-Ar), 7.50-7.30 (m, 7H, H-Ar), 5.47-5.37 (m, 1H, CHO), 4.80 (d, $\left.J_{4,5} 0.8 \mathrm{~Hz}, 1 \mathrm{H}, \mathrm{H}-4\right), 4.63$ (d, $J_{\text {gem }} 11.9 \mathrm{~Hz}, 1 \mathrm{H}$, $\mathrm{C}_{2} \mathrm{Ph}$ ), 4.57 (d, $\left.J_{\text {gem }} 11.9 \mathrm{~Hz}, 1 \mathrm{H}, \underline{\mathrm{C}}_{2} \mathrm{Ph}\right), 3.84$ (dd, $J 9.6,7.3 \mathrm{~Hz}, 1 \mathrm{H}, \mathrm{CH}_{2} \mathrm{O}$ ), 3.73 (dd, $J$ 9.6, $6.2 \mathrm{~Hz}, 1 \mathrm{H}, \mathrm{CH}_{2} \mathrm{O}$ ), 3.12-2.93 (m, 2H, H-1, H-5), 2.57-2.33 (m, 2H, 2H-cyclobutane), 2.28-2.08 (m, 2H, 2H-cyclobutane). ${ }^{13} \mathrm{C}$ NMR $\left(62.5 \mathrm{MHz}, \mathrm{CDCl}_{3}\right) \delta_{\mathrm{C}} 180.0(\mathrm{C}=\mathrm{O}), 165.1(\mathrm{C}=\mathrm{O}), 137.3$, 133.3, 129.5, 128.8, 128.4, 128.2, 127.6, 127.4 (C-Ar), 82.7 (C-4), $73.3\left(\underline{\mathrm{CH}}_{2} \mathrm{Ph}\right), 73.2(\mathrm{CHO})$, $67.3\left(\mathrm{CH}_{2} \mathrm{O}\right), 38.6,36.7$ (C-1/C-5), 24.5, 23.5 (C-6/C-7).

12c. (data extracted from an enriched sample) IR (ATR): 3063, 3031, 2954, 2923, 1770, 1718 $\mathrm{cm}^{-1} .{ }^{1} \mathrm{H}$ NMR $\left(250 \mathrm{MHz}, \mathrm{CDCl}_{3}\right) \delta_{\mathrm{H}} 8.20-7.30(\mathrm{~m}, 10 \mathrm{H}, \mathrm{H}-\mathrm{Ar}), 5.51-4.98(\mathrm{~m}, 1 \mathrm{H}, \mathrm{CHO}), 4.88$ (dd, J 8.4, 5.6 Hz, 1H, H-4), 4.70-4.40 (m, 2H, $\left.\mathrm{CH}_{2} \mathrm{Ph}\right), 3.50-4.00\left(\mathrm{~m}, 2 \mathrm{H}, \mathrm{CH}_{2} \mathrm{O}\right), 3.25-2.75$ (m, 2H, H-1, H-5), 2.64-2.31 (m, 2H, 2H-cyclobutane), 2.22-2.00 (m, 2H, 2H-cyclobutane). ${ }^{13} \mathrm{C}$ NMR $\left(62.5 \mathrm{MHz}, \mathrm{CDCl}_{3}\right) \delta_{\mathrm{C}} 180.1(\mathrm{C}=\mathrm{O}), 169.9(\mathrm{C}=\mathrm{O}), 137.1,133.0,129.9,128.4,128.4$, 128.2, 127.5, $127.4(\mathrm{C}-\mathrm{Ar}), 82.7(\mathrm{C}-4), 74.0\left(\underline{\mathrm{CH}}_{2} \mathrm{Ph}\right), 73.8(\mathrm{CHO}), 68.6\left(\mathrm{CH}_{2} \mathrm{O}\right), 39.2,37.4(\mathrm{C}-$ 1/C-5), 25.2, 24.2 (C-6/C-7).

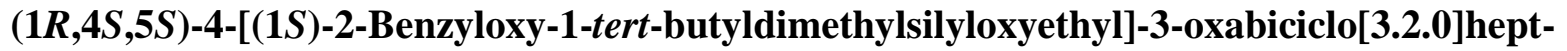
an-2-one, 11d. Following the general procedure, a solution of the furanone 5d (150 mg, 0.443 $\mathrm{mmol})$ in acetone $(65 \mathrm{~mL})$ saturated with ethylene was irradiated through a Pyrex filter for $3.5 \mathrm{~h}$. Evaporation of the solvent and purification by FCC (hexanes/EtOAc, 9:1) furnished the anti cycloadduct 11d $(94 \mathrm{mg}, 58 \%)$ as an oil: ${ }^{1} \mathrm{H}$ NMR $\left(250 \mathrm{MHz}, \mathrm{CDCl}_{3}\right) \delta_{\mathrm{H}} 7.40-7.29(\mathrm{~m}, 5 \mathrm{H}, \mathrm{H}-$ Ar), 4.58-4.50 (m, 3H, C $\left.\underline{H}_{2} \mathrm{Ph}, \mathrm{H}-4\right), 3.78$ (ddd, J 7.5, 5.3, $1.6 \mathrm{~Hz}, 1 \mathrm{H}, \mathrm{CHO}$ ), 3.61 (dd, J 9.1, 7.5 $\mathrm{Hz}, 1 \mathrm{H}, \mathrm{CH}_{2} \mathrm{O}$ ), 3.45 (dd, J 9.1, $5.3 \mathrm{~Hz}, 1 \mathrm{H}, \mathrm{CH}_{2} \mathrm{O}$ ), 3.15-3.02 (m, 1H, H-1/H-5), 3.02-2.94 (m, 1H, H-1/H-5), 2.60-2.48 (m, 1H, H-cyclobutane), 2.43-2.31 (m, 1H, H-cyclobutane), 2.21-2.07 (m, 2H, 2H-cyclobutane), 0.86 (s, 9H, $t$-Bu), 0.07 (s, 3H, SiMe), 0.05 (s, 3H, SiMe). ${ }^{13} \mathrm{C} \mathrm{NMR}$ $\left(62.5 \mathrm{MHz}, \mathrm{CDCl}_{3}\right) \delta_{\mathrm{C}} 180.8(\mathrm{C}=\mathrm{O}), 137.9,128.4,127.8,127.7(\mathrm{C}-\mathrm{Ar}), 85.1(\mathrm{C}-4), 73.6$ $\left(\underline{\mathrm{CH}}_{2} \mathrm{Ph}\right), 73.2(\mathrm{CHO}), 70.6\left(\mathrm{CH}_{2} \mathrm{O}\right), 39.4,37.1(\mathrm{C}-1 / \mathrm{C}-5), 25.7\left(\underline{\mathrm{Me}}_{3} \mathrm{C}\right), 24.8,24.0(\mathrm{C}-6 / \mathrm{C}-7)$, $17.9\left(\mathrm{Me}_{3} \underline{\mathrm{C}}\right),-4.5$ (SiMe), $-4.8(\mathrm{SiMe})$.

$(1 R, 4 S, 5 S)$-, 11e, and (1S,4S,5R)-4-[(1S)-1-Hydroxy-2-pivaloyloxyethyl]-3-oxabicyclo[3.2.0]heptan-2-one (12e).

Method A. Following the general procedure, a solution of the furanone 5e (521 mg, $2.28 \mathrm{mmol})$ in acetone $(300 \mathrm{~mL})$ saturated with ethylene was irradiated through a Pyrex filter for $7 \mathrm{~h} 15 \mathrm{~min}$. Evaporation of the solvent and purification by FCC (hexanes/EtOAc, from 4:1 to 2:1) afforded a 86:14 mixture of cycloadducts 11e and 12e (454 mg, 78\%) and staring material (55 $\mathrm{mg}, 10 \%)$. Repeated FCC (hexanes/EtOAc, 9:1) provided pure samples of compounds 11e and 12e as colorless solids.

Method B. A solution of lactone 5e (179 mg, $0.78 \mathrm{mmol})$ and (Z)-1,2-dichloroethylene $(285 \mu \mathrm{L}$, $3.75 \mathrm{mmol})$ in acetonitrile $(70 \mathrm{~mL})$ was irradiated through a quartz filter at $-20{ }^{\circ} \mathrm{C}$ for $3 \mathrm{~h}$. Evaporation of the solvent and FCC (hexanes/EtOAc, 4:1) afforded a mixture of the 
dichlorocyclobutane diastereomers. This crude product was dissolved in dry THF $(3.5 \mathrm{~mL})$ and heated up to the reflux temperature. Then, tributyltin hydride $(1.0 \mathrm{~mL}, 3.72 \mathrm{mmol})$ and a solution of AIBN (128 mg, $0.78 \mathrm{mmol}$ ) in dry THF were added dropwise under a nitrogen atmosphere. The mixture was stirred for $35 \mathrm{~min}$. Evaporation of the solvent gave a residue, which was subjected to FCC (hexanes/EtOAc, 4:1) to afford a mixture of 11e and 12e (134 $\mathrm{mg}, 67 \%$ ) in a ratio 88:12.

11e + 12e. MS (ESI) $\mathrm{m} / \mathrm{z}(\%): 279.0\left(\mathrm{M}+\mathrm{Na}^{+}, 27\right), 295.0\left(\mathrm{M}+\mathrm{K}^{+}, 100\right)$. Anal. Calcd. for $\mathrm{C}_{13} \mathrm{H}_{20} \mathrm{O}_{5}$ : C, 60.92; $\mathrm{H}, 7.87$. Found: $\mathrm{C}, 60.77 ; \mathrm{H}, 7.78 \%$.

11e. $\mathrm{mp} 94-96^{\circ} \mathrm{C}$ (EtOAc/hexanes). $[\alpha]_{\mathrm{D}}-7.6$ (c 2.0, $\left.\mathrm{CHCl}_{3}\right)$. IR (ATR): $3481,2943,1759,1704$ $\mathrm{cm}^{-1} .{ }^{1} \mathrm{H}$ NMR $\left(250 \mathrm{MHz}, \mathrm{CDCl}_{3}\right) \delta_{\mathrm{H}} 4.40(\mathrm{~d}, J 2.2 \mathrm{~Hz}, 1 \mathrm{H}, \mathrm{H}-4), 4.27(\mathrm{dd}, J 11.7,6.9 \mathrm{~Hz}, 1 \mathrm{H}$, $\mathrm{CH}_{2} \mathrm{O}$ ), 4.21 (dd, $J 11.7,4.8 \mathrm{~Hz}, 1 \mathrm{H}, \mathrm{CH}_{2} \mathrm{O}$ ), 3.83 (dddd, J 7.0, 5.2, 4.8, 2.2 Hz, 1H, CHO), 3.233.07 (m, 2H, 2H-cyclobutane), $2.72(\mathrm{~d}, J 5.2 \mathrm{~Hz}, 1 \mathrm{H}, \mathrm{OH}), 2.68-2.52$ (m, 1H, H-cyclobutane), 2.51-2.33 (m, 1H, H-cyclobutane), 2.25-2.05 (m, 2H, 2H-cyclobutane), 1.24 (s, 9H, $t$-Bu). ${ }^{13} \mathrm{C}$ NMR (62.5 MHz, $\left.\mathrm{CDCl}_{3}\right) \delta_{\mathrm{C}} 180.4(\mathrm{C}=\mathrm{O}), 178.6(\mathrm{C}=\mathrm{O}), 84.7(\mathrm{C}-4), 71.8(\mathrm{CHO}), 65.0\left(\mathrm{CH}_{2} \mathrm{O}\right)$, 38.8 (C-1), $38.6\left(\mathrm{Me}_{3} \underline{\mathrm{C}}\right), 36.9$ (C-5), 26.9 ( $\left.\underline{\mathrm{Me}}_{3} \mathrm{C}\right), 24.6,23.6$ (C-6/C-7).

12e. $\mathrm{mp} 138-140{ }^{\circ} \mathrm{C}$ (EtOAc/hexanes). $[\alpha]_{\mathrm{D}}+67.4$ (c 0.6, $\left.\mathrm{CHCl}_{3}\right)$. IR (ATR): 3510, 2975, 1761, $1708 \mathrm{~cm}^{-1} .{ }^{1} \mathrm{H}$ NMR $\left(250 \mathrm{MHz}, \mathrm{CDCl}_{3}\right) \delta_{\mathrm{H}} 4.44(\mathrm{dd}, J 7.5,5.4 \mathrm{~Hz}, 1 \mathrm{H}, \mathrm{H}-4), 4.24-4.11(\mathrm{~m}, 2 \mathrm{H}$, $\mathrm{CH}_{2} \mathrm{O}, \mathrm{CHO}$ ), 4.11-3.98 (m, 1H, $\mathrm{CH}_{2} \mathrm{O}$ ), 3.26-3.18 (m, $\left.2 \mathrm{H}, \mathrm{H}-5, \mathrm{OH}\right), 2.65-2.34$ (m, 3H, H-6, H7, H-1), 2.08-2.29 (m, 2H, H-6, H-7), 1.25 (s, 9H, $t$-Bu). ${ }^{13} \mathrm{C} \mathrm{NMR}\left(62.5 \mathrm{MHz}, \mathrm{CDCl}_{3}\right) \delta_{\mathrm{C}} 179.2$ $(\mathrm{C}=\mathrm{O}), 178.2(\mathrm{C}=\mathrm{O}), 82.4(\mathrm{C}-4), 69.5(\mathrm{CHO}), 64.2\left(\mathrm{CH}_{2} \mathrm{O}\right), 40.1(\mathrm{C}-1), 38.6\left(\mathrm{Me}_{3} \mathrm{C}\right), 36.1(\mathrm{C}-5)$, $26.9\left(\underline{\mathrm{Me}}_{3} \mathrm{C}\right), 22.9,19.6$ (C-6/C-7).

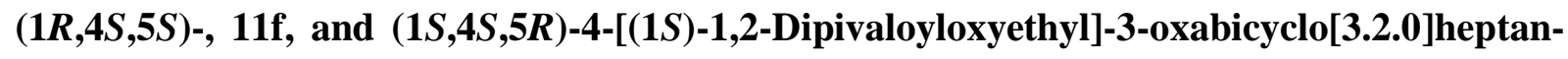
2-one (12f). Following the general procedure, a solution of the furanone $\mathbf{5 f}(510 \mathrm{mg}, 1.63 \mathrm{mmol})$ in acetone $(300 \mathrm{~mL})$ saturated with ethylene was irradiated through a Pyrex filter for $6 \mathrm{~h} 40 \mathrm{~min}$. Evaporation of the solvent and purification by FCC (hexanes/EtOAc, 4:1) afforded a 90:10 mixture of cycloadducts 11f and 12f (427 mg, 77\%). Repeated FCC (hexanes/EtOAc, 9:1) provided a pure sample of $\mathbf{1 1 f}$ as solid and enriched fractions of $\mathbf{1 2 f}$.

11f + 12f. MS (ESI) $m / z(\%): 363.2\left(\mathrm{M}+\mathrm{Na}^{+}, 54\right), 379.1\left(\mathrm{M}+\mathrm{K}^{+}, 100\right)$. Anal. Calcd. for $\mathrm{C}_{18} \mathrm{H}_{28} \mathrm{O}_{6}: \mathrm{C}, 63.51 ; \mathrm{H}, 8.29$. Found: $\mathrm{C}, 63.61 ; \mathrm{H}, 8.17 \%$.

11f: $\mathrm{mp} 79-80{ }^{\circ} \mathrm{C}$ (EtOAc/hexanes). $[\alpha]_{\mathrm{D}}-50.7$ (c 0.71, $\mathrm{CHCl}_{3}$ ). IR (ATR): 2972, 2873, 1774, $1724 \mathrm{~cm}^{-1} .{ }^{1} \mathrm{H}$ NMR $\left(250 \mathrm{MHz}, \mathrm{CDCl}_{3}\right) \delta_{\mathrm{H}} 5.20(\mathrm{ddd}, J 7.7,4.5,2.2 \mathrm{~Hz}, 1 \mathrm{H}, \mathrm{CHO}), 4.58(\mathrm{dd}, J$ $2.2,1.0 \mathrm{~Hz}, 1 \mathrm{H}, \mathrm{H}-4), 4.40\left(\mathrm{dd}, J 11.7,4.5 \mathrm{~Hz}, 1 \mathrm{H}, \mathrm{CH}_{2} \mathrm{O}\right) 4.16\left(\mathrm{dd}, J 11.7,7.7 \mathrm{~Hz}, 1 \mathrm{H}, \mathrm{CH}_{2} \mathrm{O}\right)$, 3.14-2.91 (m, 2H, H-1, H-5), 2.68-2.37 (m, 2H, 2H-cyclobutane), 2.28-2.06 (m, 2H, 2Hcyclobutane), $1.22\left(\mathrm{~s}, 9 \mathrm{H}, t\right.$-Bu), $1.21\left(\mathrm{~s}, 9 \mathrm{H}, t\right.$-Bu). ${ }^{13} \mathrm{C} \mathrm{NMR}\left(62.5 \mathrm{MHz}, \mathrm{CDCl}_{3}\right) \delta_{\mathrm{C}} 179.2$ $(\mathrm{C}=\mathrm{O}), 177.2,(\mathrm{C}=\mathrm{O}), 169.7(\mathrm{C}=\mathrm{O}), 83.3(\mathrm{C}-4), 71.8(\mathrm{CHO}), 62.4\left(\mathrm{CH}_{2} \mathrm{O}\right), 38.7\left(\mathrm{Me}_{3} \underline{\mathrm{C}}\right), 38.5$ $\left(\mathrm{Me}_{3} \mathrm{C}\right), 38.2,36.2$ (C-1/C-5), 26.8 ( $\left.\mathrm{Me}_{3} \mathrm{C}\right), 24.7,23.5$ (C-6/C-7).

12f. (data extracted from an enriched sample) IR (ATR): 2870, 2935, 2873, 1775, $1731 \mathrm{~cm}^{-1} .{ }^{1} \mathrm{H}$ NMR $\left(250 \mathrm{MHz}, \mathrm{CDCl}_{3}\right) \delta_{\mathrm{H}} 5.42-5.26(\mathrm{~m}, 1 \mathrm{H}, \mathrm{CHO}), 4.58(\mathrm{dd}, J$ 8.8, $5.2 \mathrm{~Hz}, 1 \mathrm{H}, \mathrm{H}-4), 4.37$ (dd, $\left.J 12.4,3.1 \mathrm{~Hz}, 1 \mathrm{H}, \mathrm{CH}_{2} \mathrm{O}\right), 3.94\left(\mathrm{dd}, J 12.4,5.2 \mathrm{~Hz}, 1 \mathrm{H}, \mathrm{CH}_{2} \mathrm{O}\right), 3.30-3.10$ (m, 2H, H-1, H5), 2.60-2.30 (m, 2H, 2H-cyclobutane), 2.30-2.00 (m, 2H, 2H-cyclobutane). ${ }^{13} \mathrm{C}$ NMR (62.5 
$\left.\mathrm{MHz}, \mathrm{CDCl}_{3}\right) \delta_{\mathrm{C}} 178.8(\mathrm{C}=\mathrm{O}), 177.6(\mathrm{C}=\mathrm{O}), 177.1(\mathrm{C}=\mathrm{O}), 79.6(\mathrm{C}-4), 70.5(\mathrm{CHO}), 61.7$ $\left(\mathrm{CH}_{2} \mathrm{O}\right), 39.9,36.1$ (C-1/C-5), $38.7\left(\mathrm{Me}_{3} \underline{\mathrm{C}}\right), 26.9\left(\mathrm{Me}_{3} \mathrm{C}\right), 26.8\left(\underline{\mathrm{Me}}_{3} \mathrm{C}\right), 23.0,19.8$ (C-6/C-7).

$(1 R, 4 S, 5 S)$-, $11 g$, and $(1 S, 4 S, 5 R)-4-[(1 S)$-1-Isovaleroyloxy-2-pivaloyloxyethyl]-3-oxabicyclo[3.2.0]heptan-2-one (12g). Following the general procedure, a solution of the furanone $\mathbf{5 g}$ (106 $\mathrm{mg}, 0.339 \mathrm{mmol})$ in acetone $(65 \mathrm{~mL})$ saturated with ethylene was irradiated through a Pyrex filter for $5 \mathrm{~h}$. Evaporation of the solvent and purification by FCC (from hexanes to hexanes/EtOAc, 4:1) afforded a 90:10 mixture of cycloadducts $\mathbf{1 1 g}$ and $\mathbf{1 2 g}$ (96 $\mathrm{mg}, 83 \%$ ). Repeated FCC (hexanes/EtOAc, 9:1) provided a pure sample of 11g as a solid and enriched fractions of $\mathbf{1 2} \mathrm{g}$ as a colorless oil. Both isomers slowly decompose on standing at room temperature.

11g + 12g. MS (EI) m/z (\%): $363.2\left(\mathrm{M}+\mathrm{Na}^{+}, 55\right), 379.1\left(\mathrm{M}+\mathrm{K}^{+}, 100\right)$. HRMS (FAB+): calculated for $\left[\mathrm{C}_{18} \mathrm{H}_{28} \mathrm{O}_{6}+\mathrm{H}\right]^{+}$341.1964; found 341.1969.

11g: $\mathrm{mp} 61-62{ }^{\circ} \mathrm{C}\left(\mathrm{Et}_{2} \mathrm{O} /\right.$ hexanes $) .[\alpha]_{\mathrm{D}}-50.3$ (c $2.9 \mathrm{CHCl}_{3}$ ). IR (ATR): 2959, 2936, 2906, 2870, 1760, $1731 \mathrm{~cm}^{-1} .{ }^{1} \mathrm{H}$ NMR $\left(250 \mathrm{MHz}, \mathrm{CDCl}_{3}\right) \delta_{\mathrm{H}} 5.22$ (ddd, J 7.5, 4.7, $\left.2.3 \mathrm{~Hz}, 1 \mathrm{H}, \mathrm{CHO}\right), 4.55$ $(\mathrm{d}, J 2.3,1.0 \mathrm{~Hz}, 1 \mathrm{H}, \mathrm{H}-4), 4.36\left(\mathrm{dd}, J 11.7,4.7 \mathrm{~Hz}, 1 \mathrm{H}, \mathrm{CH}_{2} \mathrm{O}\right), 4.16(\mathrm{dd}, J 11.7,7.5 \mathrm{~Hz}, 1 \mathrm{H}$, $\mathrm{CH}_{2} \mathrm{O}$ ), 3.10-2.92 (m, 2H, H-1, H-5), 2.66-2.50 (m, 1H, H-7), 2.50-2.37 (m, 1H, H-6), 2.25-2.00 (m, 5H, H-6, H-7, COC $\underline{H}_{2} \mathrm{CHMe}_{2}, \mathrm{COCH}_{2} \mathrm{CHMe}_{2}$ ), 1.20 (s, 9H, $\left.t-\mathrm{Bu}\right), 0.98$ (d, 6H, $J 6.6 \mathrm{~Hz}$, $\left.\mathrm{COCH}_{2} \mathrm{CHMe}_{2}\right) .{ }^{13} \mathrm{C} \mathrm{NMR}\left(62.5 \mathrm{MHz}, \mathrm{CDCl}_{3}\right) \delta_{\mathrm{C}} 179.3(\mathrm{C}=\mathrm{O}), 177.6(\mathrm{C}=\mathrm{O}), 171.7(\mathrm{C}=\mathrm{O})$, 82.9 (C-4), $71.6(\mathrm{CHO}), 62.2\left(\mathrm{CH}_{2} \mathrm{O}\right), 42.8\left(\mathrm{COC}_{2} \mathrm{CHMe}_{2}\right), 38.4\left(\mathrm{Me}_{3} \underline{\mathrm{C}}\right), 38.3(\mathrm{C}-1), 36.5(\mathrm{C}-$ 5), $26.8\left(\underline{\mathrm{Me}}_{3} \mathrm{C}\right), 25.3\left(\mathrm{COCH}_{2} \underline{\mathrm{CHMe}}_{2}\right), 24.6$ (C-6), 23.5 (C-7), $22.1\left(\mathrm{COCH}_{2} \mathrm{CHMe}_{2}\right)$.

12g. (data extracted from an enriched sample) IR (ATR): 2959, 2936, 2906, 2870, 1760, 1731 $\mathrm{cm}^{-1} .{ }^{1} \mathrm{H}$ NMR $\left(250 \mathrm{MHz}, \mathrm{CDCl}_{3}\right) \delta_{\mathrm{H}} 5.39$ (ddd, $J$ 7.5, 4.7, $2.7 \mathrm{~Hz}, 1 \mathrm{H}, \mathrm{CHO}$ ), 4.68 (dd, $J$ 5.7, $2.7 \mathrm{~Hz}, 1 \mathrm{H}, \mathrm{H}-4), 4.40\left(\mathrm{dd}, J 11.6,4.7 \mathrm{~Hz}, 1 \mathrm{H}, \mathrm{CH}_{2} \mathrm{O}\right), 4.20\left(\mathrm{dd}, J 11.6,7.5 \mathrm{~Hz}, 1 \mathrm{H}, \mathrm{CH}_{2} \mathrm{O}\right.$ ), 2.64-2.32 (m, 4H, H-1, H-5, CH ${ }_{2}$-cyclobutane), 2.32-1.96 (m, 5H, COC $\underline{H}_{2} \underline{\mathrm{HMe}}_{2}, \mathrm{CH}_{2}-$ cyclobutane), $1.20\left(\mathrm{~s}, 9 \mathrm{H}, t\right.$-Bu), 0.99 (d, $\left.J 6.4 \mathrm{~Hz}, 6 \mathrm{H}, \mathrm{COCH}_{2} \mathrm{CHMe}_{2}\right) .{ }^{13} \mathrm{C} \mathrm{NMR}(62.5 \mathrm{MHz}$, $\left.\mathrm{CDCl}_{3}\right) \delta_{\mathrm{C}} 177.8(\mathrm{C}=\mathrm{O}), 177.6(\mathrm{C}=\mathrm{O}), 171.8(\mathrm{C}=\mathrm{O}), 80.1(\mathrm{C}-4), 72.0(\mathrm{CHO}), 63.5\left(\mathrm{CH}_{2} \mathrm{O}\right), 43.6$ $\left(\mathrm{CO} \mathrm{H}_{2} \mathrm{CHMe}_{2}\right), 43.1$ (C-1), $38.4\left(\mathrm{Me}_{3} \underline{\mathrm{C}}\right), 36.6$ (C-5), 27.5 ( $\left.\mathrm{Me}_{3} \mathrm{C}\right), 27.0\left(\mathrm{COCH}_{2} \mathrm{CHMe}_{2}\right), 25.4$, 23.6 (C-6/C-7), $22.8\left(\mathrm{COCH}_{2} \mathrm{CHMe}_{2}\right)$.

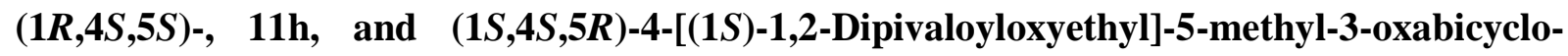
[3.2.0]heptan-2-one (12h). Following the general procedure, a solution of the furanone $\mathbf{5 h}$ (166 $\mathrm{mg}, 0.509 \mathrm{mmol})$ in acetone $(65 \mathrm{~mL})$ saturated with ethylene was irradiated through a Pyrex filter for $5 \mathrm{~h}$. Evaporation of the solvent and purification by FCC (hexanes/EtOAc, 4:1) afforded a 80:20 mixture of cycloadducts $\mathbf{1 1 h}$ and $\mathbf{1 2 h}$ (144 $\mathrm{mg}, 80 \%)$ and starting material (18 $\mathrm{mg}, 11 \%)$. Repeated FCC (hexanes/EtOAc, 9:1) provided pure samples of the two isomers $\mathbf{1 1 h}$ and $\mathbf{1 2 h}$ as solids.

11h + 12h. HRMS $m / z(F A B+)$ : calculated for $\left[\mathrm{C}_{19} \mathrm{H}_{30} \mathrm{O}_{6}+\mathrm{H}\right]^{+}$377.1935; found 377.1934

11h. Mp 76-78 ${ }^{\circ} \mathrm{C}$ (EtOAc/hexanes). $[\alpha]_{\mathrm{D}}-28.3$ (c 1.0, $\mathrm{CHCl}_{3}$ ). IR (ATR): 2970, 2940, 2911, 2873, 1766, 1735, $1724 \mathrm{~cm}^{-1} .{ }^{1} \mathrm{H}$ NMR $\left(400 \mathrm{MHz}, \mathrm{CDCl}_{3}\right) \delta_{\mathrm{H}} 5.18$ (ddd, J 7.4, 5.1, $0.6 \mathrm{~Hz}, 1 \mathrm{H}$, $\mathrm{CHO}$ ), 4.36-4.28 (m, 2H, H-4, $\left.1 \mathrm{H}-\mathrm{CH}_{2} \mathrm{O}\right), 4.12$ (dd, $J$ 11.4, 7.4 Hz, $1 \mathrm{H}, \mathrm{CH}_{2} \mathrm{O}$ ), 2.65-2-60 (m, 1H, H-cyclobutane), 2.60-2.46 (m, 1H, H-cyclobutane), 2.38-2.28 (m, 1H, H-cyclobutane), 2.08- 
2-02 (m, 1H, H-cyclobutane), 1.96-1.85 (m, 1H, H-cyclobutane), 1.24 (s, 3H, Me), 1.17 (s, 9H, $t$ $\mathrm{Bu}), 1.16\left(\mathrm{~s}, 9 \mathrm{H}, t\right.$-Bu). ${ }^{13} \mathrm{C}$ NMR $\left(100 \mathrm{MHz}, \mathrm{CDCl}_{3}\right) \delta_{\mathrm{C}} 179.6(\mathrm{C}=\mathrm{O}), 177.9(\mathrm{C}=\mathrm{O}), 176.7$ $(\mathrm{C}=\mathrm{O}), 84.7(\mathrm{C}-4), 69.6(\mathrm{CHO}), 63.1\left(\mathrm{CH}_{2} \mathrm{O}\right), 43.4(\mathrm{C}-1), 43.3(\mathrm{C}-5), 38.8\left(\mathrm{Me}_{3} \underline{\mathrm{C}}\right), 38.7\left(\mathrm{Me}_{3} \underline{\mathrm{C}}\right)$, 32.6 (C-6/C-7), 27.1 ( $\left.\mathrm{Me}_{3} \mathrm{C}\right), 27.0\left(\underline{\mathrm{Me}}_{3} \mathrm{C}\right), 21.5$ (C-6/C-7), $17.5(\mathrm{Me})$.

12h. Mp 97-99 ${ }^{\circ} \mathrm{C}$ (EtOAc/hexanes). $[\alpha]_{\mathrm{D}}+67.5$ (c 0.4, $\mathrm{CHCl}_{3}$ ). IR (ATR): 2960, 2920, 2873, 2851, 1777, $1722 \mathrm{~cm}^{-1} .{ }^{1} \mathrm{H}$ NMR $\left(400 \mathrm{MHz}, \mathrm{CDCl}_{3}\right) \delta_{\mathrm{H}} 5.33$ (ddd, J 7.7, 4.8, 3.0 Hz, 1H, CHO), 4.46 (dd, $J$ 12.4, $\left.3.0 \mathrm{~Hz}, 1 \mathrm{H}, \mathrm{CH}_{2} \mathrm{O}\right), 4.29$ (d, J 7.7 Hz, 1H, H-4), 3.83 (dd, J 12.4, $4.8 \mathrm{~Hz}, 1 \mathrm{H}$, $\mathrm{CH}_{2} \mathrm{O}$ ), 2.77-2.73 (m, 1H, H-cyclobutane), 2.58-2.43 (m, 2H, H-cyclobutane), 2.12-2.03 (m, 1H, H-cyclobutane), 1.82-1.73 (m, 1H, H-cyclobutane), 1.42 (s, 3H, Me), 1.22 (s, 9H, $t$-Bu), 1.20 (s, $9 \mathrm{H}, t$-Bu $).{ }^{13} \mathrm{C}$ NMR $\left(100 \mathrm{MHz}, \mathrm{CDCl}_{3}\right) \delta_{\mathrm{C}} 178.6(\mathrm{C}=\mathrm{O}), 177.7(\mathrm{C}=\mathrm{O}), 177.3(\mathrm{C}=\mathrm{O}), 84.6(\mathrm{C}-4)$, $70.3(\mathrm{CHO}), 62.0\left(\mathrm{CH}_{2} \mathrm{O}\right), 45.1(\mathrm{C}-1), 43.0(\mathrm{C}-5), 38.8\left(\mathrm{Me}_{3} \underline{\mathrm{C}}\right), 29.7\left(\mathrm{Me}_{3} \underline{\mathrm{C}}\right), 27.1\left(\underline{\mathrm{Me}}_{3} \mathrm{C}\right), 27.0$ $\left(\underline{\mathrm{Me}}_{3} \mathrm{C}\right), 25.7$ (C-6/C-7), $22.0(\mathrm{Me}), 20.4$ (C-6/C-7).

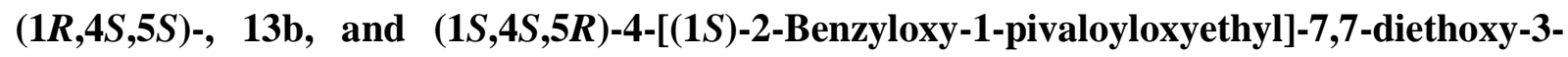
oxabicyclo[3.2.0]heptan-2-one (14b). A solution of the lactone $5 \mathbf{b}(506 \mathrm{mg}, 1.58 \mathrm{mmol})$ and 1,1-diethoxyethylene $(2.1 \mathrm{~mL}, 15.9 \mathrm{mmol})$ in acetonitrile $(300 \mathrm{~mL})$ was irradiated though a quartz filter for $2 \mathrm{~h}$. Evaporation of the solvent and FCC (hexanes/EtOAc, 9:1) afforded a 79:21 mixture of 13b and 14b (370 mg, 54\%). Repeated FCC (hexanes/EtOAc, 9:1) provided enriched fractions of $13 \mathrm{~b}$ and $\mathbf{1 4 b}$ as oils.

When the irradiation of lactone $\mathbf{5 b}(102 \mathrm{mg}, 0.32 \mathrm{mmol})$ and 1,1-diethoxyethylene ( $420 \mu \mathrm{L}, 3.2$ $\mathrm{mmol})$ was performed through a quartz filter in diethyl ether $(70 \mathrm{~mL})$ for $5 \mathrm{~h}$, purification of the crude material by FCC (hexanes/EtOAc, 9:1), afforded a 76:24 mixture of 13b and 14b (62 mg, $44 \%)$.

When the irradiation of lactone $\mathbf{5 b}(146 \mathrm{mg}, 0.46 \mathrm{mmol})$ and 1,1-diethoxyethylene ( $600 \mu \mathrm{L}, 4.5$ mmol) was performed through a quartz filter in hexane $(70 \mathrm{~mL})$ for $2 \mathrm{~h}$, purification of the crude material by FCC (hexanes/EtOAc, 9:1) afforded a 79:21 mixture of 13b and 14b (122 mg, 61\%).

13b + 14b. MS (ESI) $m / z(\%): 457.2\left(\mathrm{M}+\mathrm{H}^{+}, 100\right)$. HRMS $(\mathrm{m} / z \mathrm{FAB}+)$ : calculated for $\left[\mathrm{C}_{24} \mathrm{H}_{34} \mathrm{O}_{7}\right.$ $+\mathrm{Na}]^{+}$457.2197; found 457.2199.

13b. (data extracted from an enriched sample) IR (ATR): 2975, 2931, 1775, $1731 \mathrm{~cm}^{-1}$. ${ }^{1} \mathrm{H}$ NMR $\left(360 \mathrm{MHz}, \mathrm{CDCl}_{3}\right) \delta_{\mathrm{H}}$ 7.45-7.29 (m, 5H, H-Ar), 5.17-5.10 (m, 2H, H-4, CHO), 4.58 (d, $J_{\text {gem }} 11.9$ $\left.\mathrm{Hz}, 1 \mathrm{H}, \mathrm{C}_{2} \mathrm{Ph}\right), 4.54$ (d, $\left.J_{\text {gem }} 11.9 \mathrm{~Hz}, 1 \mathrm{H}, \mathrm{C}_{2} \mathrm{Ph}\right), 3.70\left(\mathrm{dd}, J 11.9,6.6 \mathrm{~Hz}, 1 \mathrm{H}, \mathrm{CH}_{2} \mathrm{O}\right), 3.64$ (dd, $J 11.9,6.3 \mathrm{~Hz}, 1 \mathrm{H}, \mathrm{CH}_{2} \mathrm{O}$ ), 3.47-3.27 (m, 4H, $2 \times \mathrm{OCH}_{2} \mathrm{CH}_{3}$ ), 3.03-2.89 (m, 2H, H-1, H-5), 2.59 (ddd, $\left.J_{\text {gem }} 13.5,9.6,2.2 \mathrm{~Hz}, 1 \mathrm{H}, \mathrm{H}-7\right), 2.44$ (dd, $\left.J_{\text {gem }} 13.5,4.0 \mathrm{~Hz}, \mathrm{H}-7\right), 1.20$ (s, 15H, $2 \times$ $\mathrm{OCH}_{2} \underline{\mathrm{CH}}_{3}, t$-Bu). ${ }^{13} \mathrm{C} \mathrm{NMR}\left(62.5 \mathrm{MHz}, \mathrm{CDCl}_{3}\right) \delta_{\mathrm{C}} 178.9(\mathrm{C}=\mathrm{O}), 177.7(\mathrm{C}=\mathrm{O}), 137.7,128.4$, 127.8, 127.7 (C-Ar), 99.0 (C-6), 76.7 (C-4), $73.5\left(\underline{\mathrm{CH}}_{2} \mathrm{Ph}\right), 72.7(\mathrm{CHO}), 68.1\left(\mathrm{CH}_{2} \mathrm{O}\right), 56.8,56.6$ $\left(\mathrm{OCH}_{2} \mathrm{CH}_{3}\right), 46.8$ (C-5), $39.0\left(\mathrm{Me}_{3} \underline{\mathrm{C}}\right), 36.7$ (C-7), 30.9 (C-1), $27.1 \quad\left(\underline{\mathrm{Me}}_{3} \mathrm{C}\right), 15.0,14.9$ $\left(\mathrm{OCH}_{2} \underline{\mathrm{CH}_{3}}\right)$.

14b. (data extracted from an enriched sample) IR (ATR): 2975, 2930, 2918, 1773, $1727 \mathrm{~cm}^{-1} .{ }^{1} \mathrm{H}$ NMR (360 MHz, $\left.\mathrm{CDCl}_{3}\right) \delta_{\mathrm{H}}$ 7.40-7.26 (m, 5H, H-Ar), 5.90 (ddd, J 9.5, 3.3, $2.0 \mathrm{~Hz}, 1 \mathrm{H}, \mathrm{CHO}$ ), 4.88 (dd, J 9.5, $6.6 \mathrm{~Hz}, 1 \mathrm{H}, \mathrm{H}-4), 4.59$ (d, $\left.J_{\text {gem }} 12.1 \mathrm{~Hz}, 1 \mathrm{H}, \mathrm{C}_{2} \mathrm{Ph}\right), 4.46$ (d, $J_{\text {gem }} 12.1 \mathrm{~Hz}, 1 \mathrm{H}$, $\left.\mathrm{C}_{2} \mathrm{Ph}\right), 3.92\left(\mathrm{dd}, J 11.5,3.3 \mathrm{~Hz}, 1 \mathrm{H}, \mathrm{CH}_{2} \mathrm{O}\right), 3.64\left(\mathrm{dd}, J 11.5,3.3 \mathrm{~Hz}, 1 \mathrm{H}, \mathrm{CH}_{2} \mathrm{O}\right), 3.40$ (q, $J 7.1$ 
$\left.\mathrm{Hz}, 2 \mathrm{H}, \mathrm{OC}_{2} \mathrm{CH}_{3}\right), 3.35-3.27\left(\mathrm{~m}, 1 \mathrm{H}, \mathrm{OC}_{2} \mathrm{CH}_{3}\right), 3.24-3.07$ (m, $\left.3 \mathrm{H}, \mathrm{OC}_{2} \mathrm{CH}_{3}, \mathrm{H}-1, \mathrm{H}-5\right)$, 2.59-2.44 (m, 2H, 2H-7), $1.26\left(\mathrm{~s}, 9 \mathrm{H}, t\right.$-Bu), $1.23\left(\mathrm{t}, J 7.0 \mathrm{~Hz}, 3 \mathrm{H}, \mathrm{OCH}_{2} \mathrm{CH}_{3}\right), 1.13(\mathrm{t}, J 7.0 \mathrm{~Hz}$, $\left.3 \mathrm{H}, \mathrm{OCH}_{2} \mathrm{CH}_{3}\right) .{ }^{13} \mathrm{C} \mathrm{NMR}\left(90 \mathrm{MHz}, \mathrm{CDCl}_{3}\right) \delta_{\mathrm{C}} 178.6(\mathrm{C}=\mathrm{O}), 177.7(\mathrm{C}=\mathrm{O}), 137.8,128.5,127.9$, 127.7 (C-Ar), $101.4(\mathrm{C}-6), 80.8(\mathrm{C}-4), 73.5\left(\underline{\mathrm{CH}}_{2} \mathrm{Ph}\right), 72.4(\mathrm{CHO}), 69.0\left(\mathrm{CH}_{2} \mathrm{O}\right), 57.0,56.4$ $\left(\mathrm{OCH}_{2} \mathrm{CH}_{3}\right), 45.7$ (C-5), $38.8\left(\mathrm{Me}_{3} \underline{\mathrm{C}}\right), 36.6$ (C-7), 32.2 (C-1), $27.1\left(\mathrm{Me}_{3} \mathrm{C}\right), 15.1,15.0$ $\left(\mathrm{OCH}_{2} \mathrm{CH}_{3}\right)$.

$(1 R, 4 S, 5 S)$-, 13f, and $(1 S, 4 S, 5 R)-7,7-D i e t h o x y-4-[(1 S)-1,2$-dipivaloyloxyethyl]-3-oxabicyclo[3.2.0]heptan-2-one (14f) and (1R,4S,5S)-6,6-diethoxy-4-[(1S)-1,2-dipivaloyloxyethyl]-3-oxabicyclo[3.2.0] heptan-2-one (15f). A solution of the lactone $\mathbf{5 f}(100 \mathrm{mg}, 0.32 \mathrm{mmol})$ and 1,1diethoxyethylene $(420 \mu \mathrm{L}, 3.2 \mathrm{mmol})$ in acetonitrile $(70 \mathrm{~mL})$ was irradiated though a quartz filter for 3.5 h. Evaporation of the solvent and FCC (hexanes/EtOAc, 9:1) afforded a 64:16:20 mixture of 13f, 14f and 15f (121 mg, 81\%). Repeated FCC (hexanes/EtOAc, 9:1) provided enriched fractions of $\mathbf{1 3 f}, \mathbf{1 4 f}$ and $\mathbf{1 5 f}$ as oils.

When the irradiation of lactone $\mathbf{5 f}(108 \mathrm{mg}, 0.35 \mathrm{mmol})$ and 1,1-diethoxyethylene (455 $\mu \mathrm{L}, 3.5$ mmol) was performed through a quartz filter in diethyl ether $(70 \mathrm{~mL})$ for $2.5 \mathrm{~h}$, purification of the crude material by FCC (hexanes/EtOAc, 9:1) afforded a 75:21:4 mixture of 13f, $14 \mathbf{f}$ and $\mathbf{1 5 f}$ (61 mg, 41\%).

When the irradiation of lactone $\mathbf{5 f}(113 \mathrm{mg}, 0.36 \mathrm{mmol})$ and 1,1-diethoxyethylene (480 $\mu \mathrm{L}, 3.6$ mmol) was performed through a quartz filter in hexane $(70 \mathrm{~mL})$ for $4.5 \mathrm{~h}$, purification of the crude material by FCC (hexanes/EtOAc, 9:1) afforded a 75:21:4 mixture of 13f, 14f and 15f (115 $\mathrm{mg}, 74 \%)$.

13f $+\mathbf{1 4 f}+$ 15f. HRMS $(\mathrm{FAB}+)$ : (isomers mixture): calculated for $\left[\mathrm{C}_{22} \mathrm{H}_{36} \mathrm{O}_{8}+\mathrm{Na}\right]^{+}$451.2302; found 451.2295 .

13f. (data extracted from an enriched sample) IR (ATR): 2974, 2928, 1779, $1736 \mathrm{~cm}^{-1}$. ${ }^{1} \mathrm{H}$ NMR $\left(360 \mathrm{MHz}, \mathrm{CDCl}_{3}\right) \delta_{\mathrm{H}} 5.22$ (ddd, J 8.0, 4.2, $2.1 \mathrm{~Hz}, 1 \mathrm{H}, \mathrm{CHO}$ ), 5.03 (broad s, 1H, H-4), 4.44 (dd, $\left.J 11.8,4.2 \mathrm{~Hz}, 1 \mathrm{H}, \mathrm{CH}_{2} \mathrm{O}\right), 4.15\left(\mathrm{dd}, J 11.8,8.0 \mathrm{~Hz}, 1 \mathrm{H}, \mathrm{CH}_{2} \mathrm{O}\right), 3.60-3.20(\mathrm{~m}, 4 \mathrm{H}, 2 \times$ $\mathrm{OC}_{2} \mathrm{CH}_{3}$ ), 3.00-2.90 (m, 2H, 2H-cyclobutane), 2.70-2.55 (m, 1H, H-cyclobutane), 2.50-2.40 (m, 1H, H-cyclobutane), 1.40-1.10 (m, 24H, $2 \times \mathrm{OCH}_{2} \mathrm{C}_{3}, 2 \times t$-Bu). ${ }^{13} \mathrm{C}$ NMR $(62.5 \mathrm{MHz}$, $\left.\mathrm{CDCl}_{3}\right) \delta_{\mathrm{C}} 178.1(\mathrm{C}=\mathrm{O}), 177.6(\mathrm{C}=\mathrm{O}), 177.2(\mathrm{C}=\mathrm{O}), 98.4(\mathrm{C}-6), 77.3(\mathrm{C}-4), 71.7(\mathrm{CHO}), 62.5$ $\left(\mathrm{CH}_{2} \mathrm{O}\right), 56.5\left(\mathrm{OCL}_{2} \mathrm{CH}_{3}\right), 56.4\left(\mathrm{OCH}_{2} \mathrm{CH}_{3}\right), 46.3(\mathrm{C}-5), 38.7\left(\mathrm{Me}_{3} \underline{\mathrm{C}}\right), 38.4\left(\mathrm{Me}_{3} \underline{\mathrm{C}}\right), 36.5(\mathrm{C}-7)$, 30.4 (C-1), $26.8\left(\underline{\mathrm{Me}}_{3} \mathrm{C}\right), 14.7\left(\mathrm{OCH}_{2} \underline{\mathrm{CH}}_{3}\right), 14.6\left(\mathrm{OCH}_{2} \underline{\mathrm{CH}}_{3}\right)$.

14f. (data extracted from an enriched sample) IR (ATR): 2976, 2935, 1781, $1735 \mathrm{~cm}^{-1} .{ }^{1} \mathrm{H}$ NMR $\left(360 \mathrm{MHz}, \mathrm{CDCl}_{3}\right) \delta_{\mathrm{H}} 5.96(\mathrm{dt}, J 9.5,2.8 \mathrm{~Hz}, 1 \mathrm{H}, \mathrm{CHO}), 4.70(\mathrm{dd}, J 9.5,6.6 \mathrm{~Hz}, 1 \mathrm{H}, \mathrm{H}-4), 4.48$ $\left(\mathrm{dd}, J 11.6,2.3 \mathrm{~Hz}, 1 \mathrm{H}, \mathrm{CH}_{2} \mathrm{O}\right), 4.32\left(\mathrm{dd}, J 11.6,3.3 \mathrm{~Hz}, 1 \mathrm{H}, \mathrm{CH}_{2} \mathrm{O}\right), 3.55-3.30(\mathrm{~m}, 4 \mathrm{H}, 2 \times$ $\left.\mathrm{OC}_{2} \mathrm{CH}_{3}\right), 3.30-3.05(\mathrm{~m}, 2 \mathrm{H}, \mathrm{H}-5, \mathrm{H}-7), 2.65-2.40(\mathrm{~m}, 2 \mathrm{H}, \mathrm{H}-1, \mathrm{H}-7), 1.21$ (s, 24H, $2 \times t-\mathrm{Bu}, 2$ $\left.\times \mathrm{OCH}_{2} \mathrm{C}_{3}\right) .{ }^{13} \mathrm{C}$ NMR $\left(62.5 \mathrm{MHz}, \mathrm{CDCl}_{3}\right) \delta_{\mathrm{C}} 178.6(\mathrm{C}=\mathrm{O}), 178.3(\mathrm{C}=\mathrm{O}), 177.7(\mathrm{C}=\mathrm{O}), 101.5$ (C-6), 80.7 (C-4), $71.5(\mathrm{CHO}), 62.8\left(\mathrm{CH}_{2} \mathrm{O}\right), 57.5\left(\mathrm{OCH}_{2} \mathrm{CH}_{3}\right), 57.0\left(\mathrm{OCH}_{2} \mathrm{CH}_{3}\right), 45.9(\mathrm{C}-5)$, $39.2\left(\mathrm{Me}_{3} \mathrm{C}\right), 37.3(\mathrm{C}-7), 32.5(\mathrm{C}-1), 27.6\left(\underline{\mathrm{Me}}_{3} \mathrm{C}\right), 27.4\left(\mathrm{Me}_{3} \mathrm{C}\right), 15.4\left(\mathrm{OCH}_{2} \mathrm{C}_{3}\right)$.

15f. (data extracted from an enriched sample) IR (ATR): 2974, 2928, 1778, 1730, $1480 \mathrm{~cm}^{-1}$. ${ }^{1} \mathrm{H}$ NMR (250 MHz, $\left.\mathrm{CDCl}_{3}\right) \delta_{\mathrm{H}} 5.20(\mathrm{ddd}, J 7.5,4.4,2.1 \mathrm{~Hz}, 1 \mathrm{H}, \mathrm{CHO}), 4.56$ (broad s, 1H, H-4), 
$4.36\left(\mathrm{dd}, J 11.8,4.4 \mathrm{~Hz}, 1 \mathrm{H}, \mathrm{CH}_{2} \mathrm{O}\right), 4.13\left(\mathrm{dd}, J 11.8,7.5 \mathrm{~Hz}, 1 \mathrm{H}, \mathrm{CH}_{2} \mathrm{O}\right), 3.64-3.34(\mathrm{~m}, 4 \mathrm{H}, 2 \times$ $\mathrm{OC}_{2} \mathrm{CH}_{3}$ ), 3.34-3.28 (ddd, $J$ 6.9, 3.6, $0.8 \mathrm{~Hz}, 1 \mathrm{H}, \mathrm{H}-1$ ), 2.72-2.56 (m, 1H, H-5), 2.26-2.13 (m, $1 \mathrm{H}, \mathrm{H}-6), 1.40-1.10\left(\mathrm{~m}, 24 \mathrm{H}, 2 \times \mathrm{OCH}_{2} \mathrm{CH}_{3}, 2 \times t-\mathrm{Bu}\right) .{ }^{13} \mathrm{C} \mathrm{NMR}\left(62.5 \mathrm{MHz}, \mathrm{CDCl}_{3}\right) \delta_{\mathrm{C}} 172.9$ $(\mathrm{C}=\mathrm{O}), 172.4(\mathrm{C}=\mathrm{O}), 168.2(\mathrm{C}=\mathrm{O}), 94.2(\mathrm{C}-7), 78.3(\mathrm{C}-4), 66.9(\mathrm{CHO}), 57.7\left(\mathrm{CH}_{2} \mathrm{O}\right), 52.6$ $\left(\mathrm{OCH}_{2} \mathrm{CH}_{3}\right), 52.1\left(\mathrm{OCLH}_{2} \mathrm{CH}_{3}\right), 45.6(\mathrm{C}-1), 33.9(\mathrm{C}-6), 33.7\left(\mathrm{Me}_{3} \underline{\mathrm{C}}\right), 33.3\left(\mathrm{Me}_{3} \underline{\mathrm{C}}\right), 23.8(\mathrm{C}-5)$, $22.1\left(\underline{\mathrm{Me}_{3} \mathrm{C}}\right), 10.0\left(\mathrm{OCH}_{2} \underline{\mathrm{CH}}_{3}\right)$.

\section{Acknowledgements}

We acknowledge the Spanish Dirección General de Investigación for financial support (project CTQ2010-15380) and for a grant (to J. R. C.-G.).

\section{References}

1. De Souza, M. V. N. Mini-Rev. Org. Chem. 2005, 2, 139. http://dx.doi.org/10.1016/S0040-4039(02)02528-5

2. Nouri, D. H.; Tantillo, D. J. Curr. Org. Chem. 2006, 10, 2055. http://dx.doi.org/10.2174/138527206778742678

3. Sergeiko, A.; Poroikov, V. V.; Hanus, L. O.; Dembitsky, V. M. Open Med. Chem. J. 2008, 2, 26.

http://dx.doi.org/10.2174/1874104500802010026

4. Hansen, T. V.; Stenstrom, Y. In Organic Synthesis: Theory and Applications; Hudlicky, T. Ed.; Elsevier New York, 2001, Vol. 5, p 1. http://dx.doi.org/10.1016/S1047-773X(01)80002-0

5. Alibés, R.; Bourdelande, J. L.; Font, J.; Parella, T. Tetrahedron 1996, 52, 1279. http://dx.doi.org/10.1016/0040-4020(95)00958-2

6. de March, P.; Figueredo, M.; Font, J.; Raya, J. Org. Lett. 2000, 2, 163. http://dx.doi.org/10.1021/o1991261k

7. Alibés, R.; de March, P.; Figueredo, M.; Font, J.; Racamonde, M.; Parella, T. Org. Lett. 2004, 6, 1449.

http://dx.doi.org/10.1021/o10497032

8. Racamonde, M.; Alibés, R.; Figueredo, M.; de March, P.; Font, J. J. Org. Chem. 2008, 73, 5944. http://dx.doi.org/10.1021/j0800970u

9. Parés, S.; Alibés, R.; Figueredo, M.; Font, J.; Parella, T. Eur. J. Org. Chem. 2012, 1404. http://dx.doi.org/10.1002/ejoc.201101614

10. Pérez, L.; Alibés, R.; de March, P.; Busqué, F.; Figueredo, M.; Font , J. J. Org. Chem. 2013, 78, 4483.

http://dx.doi.org/10.1021/jo400487y 
11. Alibés, R.; Alvarez-Larena, A.; de March, P.; Figueredo, M.; Font, J.; Parella, T.; Rustullet, A. Org. Lett. 2006, 8, 491. http://dx.doi.org/10.1021/o1052794y

12. Rustullet, A.; Alibés, R.; de March, P.; Figueredo, M.; Font, J. Org. Lett. 2007, 9, 2827. http://dx.doi.org/10.1021/o10710616

13. Flores, R.; Rustullet, A.; Alibés, R.; Álvarez-Larena, A.; de March, P.; Figueredo, M.; Font, J. J. Org. Chem. 2011, 76, 5369.

14. Alibes, R.; Font, J. Afinidad 2007, 64, 189.

15. Lee-Ruff, E.; Mladenova, G. Chem. Rev. 2003, 103, 1449.

http://dx.doi.org/10.1021/cr010013a

16. Namyslo, J. C.; Kaufmann, D. Chem. Rev. 2003, 103, 1485.

http://dx.doi.org/10.1021/cr010010y

17. Hoffmann, N. Chem. Rev. 2008, 108, 1052. http://dx.doi.org/10.1021/cr0680336

18. Iriondo-Alberdi, J.; Greaney, M. F. Eur. J. Org. Chem. 2007, 4801. http://dx.doi.org/10.1002/ejoc.200700239

19. Tomioka, K.; Kawasaki, H.; Iiataka, Y.; Koga, K. Tetrahedron Lett. 1985, 26, 903. http://dx.doi.org/10.1016/S0040-4039(00)61960-3

20. Tomioka, K.; Cho, Y.-S.; Sato, F.; Koga, K. J. Org. Chem. 1988, 53, 4094. http://dx.doi.org/10.1021/jo00252a039

21. Alibés, R.; Bourdelande, J. L.; Font, J. Tetrahedron: Asymmetry 1991, 2, 1391. http://dx.doi.org/10.1016/S0957-4166(00)80035-X

22. Gregori, A.; Alibés, R.; Bourdelande, J. L.; Font, J. Tetrahedron Lett. 1998, 39, 6961. http://dx.doi.org/10.1016/S0040-4039(98)01476-2

23. de March, P.; Figueredo, M.; Font, J.; Raya, J.; Alvarez-Larena, A.; Piniella, J. F. J. Org. Chem. 2003, 68, 2437.

http://dx.doi.org/10.1021/jo026705w

24. Mukaiyama, T.; Suzuki, K.; Yamada, T.; Tabusa, F. Tetrahedron 1990, 46, 265. http://dx.doi.org/10.1016/S0040-4020(01)97597-0

25. Mash, E. A.; Nelson, K. A.; Van Deusen, S.; Hemperly, S. B. Org. Synth. 1989, 68, 92.

26. Ogawa, H.; Amano, M.; Chichara, T. Chem. Commun. 1998, 495. http://dx.doi.org/10.1039/a707753j

27. Sánchez-Sancho, F.; Valverde, S.; Herradón, B. Tetrahedron: Asymmetry 1996, 7, 3209. http://dx.doi.org/10.1016/0957-4166(96)00424-7

28. Alibés, R.; de March, P.; Figueredo, M.; Font, J.; Racamonde, M.; Rustullet, A.; AlvarezLarena, A.; Piniella, J. F.; Parella, T. Tetrahedron Lett. 2003, 44, 69. http://dx.doi.org/10.1016/S0040-4039(02)02528-5

29. Rustullet, A.; Racamonde, M.; Alibés, R.; de March, P.; Figueredo, M.; Font, J. Tetrahedron 2008, 64, 9442.

http://dx.doi.org/10.1016/j.tet.2008.07.082 